\title{
Multifaceted Study of the Interactions between CPO-27-Ni and Polyurethane and Their Impact on Nitric Oxide Release Performance
}

\author{
Simon M. Vornholt, Morven J. Duncan, Stewart J. Warrender, Rocio Semino, Naseem A. Ramsahye, \\ Guillaume Maurin, Martin W. Smith, Jin-Chong Tan, David N. Miller, and Russell E. Morris*
}

Cite This: ACS Appl. Mater. Interfaces 2020, 12, 58263-58276

Read Online

\section{ACCESS | \\ Џlll Metrics \& More \\ Article Recommendations \\ Supporting Information}

ABSTRACT: A multifaceted study involving focused ion beam scanning electron microscopy techniques, mechanical analysis, water adsorption measurements, and molecular simulations is employed to rationalize the nitric oxide release performance of polyurethane films containing 5, 10, 20, and 40 wt $\%$ of the metal-organic framework (MOF) CPO-27-Ni. The polymer and the MOF are first demonstrated to exhibit excellent compatibility. This is reflected in the even distribution and encapsulation of large wt \% MOF loadings throughout the full thickness of the films and by the rather minimal influence of the MOF on the mechanical properties of the polymer at low wt \%. The NO release efficiency of the MOF is attenuated by the polymer and

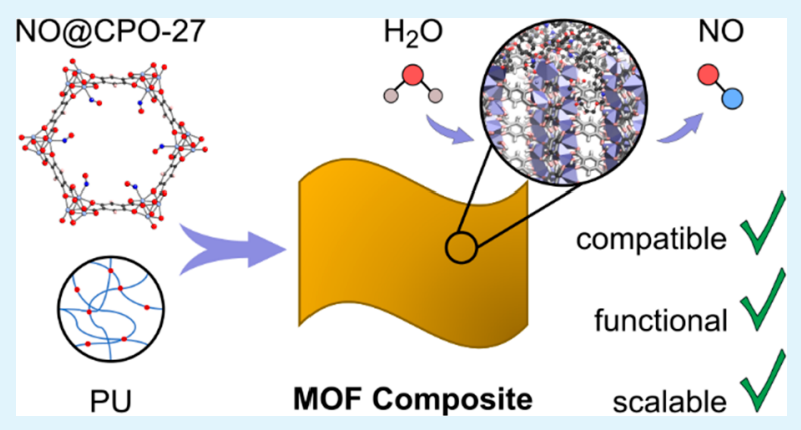
found to depend on wt \% of MOF loading. The formation of a fully connected network of MOF agglomerates within the films at higher wt \% is proposed to contribute to a more complex guest transport in these formulations, resulting in a reduction of NO release efficiency and film ductility. An optimum MOF loading of 10 wt \% is identified for maximizing NO release without adversely impacting the polymer properties. Bactericidal efficacy of released NO from the films is demonstrated against Pseudomonas aeruginosa, with a $>8 \log _{10}$ reduction in cell density observed after a contact period of $24 \mathrm{~h}$.

KEYWORDS: metal-organic frameworks, mixed-matrix membranes, composite materials, FIB-SEM, nitric oxide, medical applications

\section{INTRODUCTION}

Nitric oxide (NO) has received much attention in recent years because of its importance in many physiological and pathological pathways in the human body. Low concentrations $(\mathrm{pM}-\mu \mathrm{M})$ of $\mathrm{NO}$ are naturally produced in human cells by nitric oxide synthase enzymes. In this concentration range, NO plays an important role as a signaling molecule in promoting wound healing, ${ }^{1}$ in preventing blood clots (antithrombosis), and in controlling blood pressure. ${ }^{3}$ Furthermore, NO has also been highlighted as a potent antimicrobial agent. ${ }^{4,5}$ Exogenous delivery of NO could therefore be very beneficial in a healthcare setting. ${ }^{6,7}$ An example of where such delivery could be highly advantageous is in the fight against Healthcare Associated Infection (or HAIs). In the US alone, they contribute to 98,000 fatalities each year and bring a significant financial burden ( $\$ 28-\$ 45$ billion) as a result of elongated hospitalization periods and associated additional care. ${ }^{1,2}$ Urinary tract infections (UTIs) are the most common type of HAIs and represent $\sim 36 \%$ of all infections. Of these, $80 \%$ are associated with the use of a urinary catheter (infections known as catheter-associated UTIs or CAUTIs). Even shortterm use of such devices (e.g., during surgical procedures conducted under anesthesia) carries a risk of infection. ${ }^{8}$ Releasing NO locally from the surface of these devices in concentrations deemed to elicit an antibacterial effect has the potential to reduce such infections.

Metal-organic frameworks (MOFs) are a prominent class of (nano-)porous materials that are composed of metal ions or clusters linked via polydentate organic molecules. Their exceptionally high porosity and surface area make them potentially applicable to a wide range of innovative fields such as gas storage $^{9-11}$ and separation, ${ }^{12,13}$ catalysis, ${ }^{14,15}$ sensing, ${ }^{16,17}$ and drug delivery. ${ }^{18-22}$ Several research groups have shown that MOFs are very effective for the storage, release, and/or catalytic production of NO. ${ }^{11,23-26}$ We have shown that MOFs with coordinatively unsaturated sites (CUSs) such as the CPO-27 (MOF-74) family display promise as NO storage/release agents, capable of releasing their payloads when exposed to moisture. ${ }^{11,23}$ This family of MOFs is particularly suitable in this regard due to their high density of $\mathrm{CUSs}^{27}$ and stability toward adsorbed water and

Received: October 6, 2020

Accepted: November 30, 2020

Published: December 16, 2020 
toxic gases. ${ }^{28}$ Although CPO-27 can be prepared with many different metal species in its framework, ${ }^{29-31}$ the $\mathrm{Ni}$ analogue affords the most efficient adsorption/storage/release cycle profile combined with the highest concentration of $\mathrm{NO}$ released $(\sim 7 \mathrm{mmol} / \mathrm{g}) .{ }^{11}$ One of the strengths of MOFs as NO delivery agents compared to the likes of S-nitrosothiols and NONOates is their ability to release larger concentrations of NO. ${ }^{32,33}$ Insufficient delivery by other agents is often a factor that results in low, ineffective, and/or slow antimicrobial efficacy and necessitates the combination of NO with other agents, for example, silver nanoparticles. ${ }^{34-37}$ Furthermore, the concentration of NO that is released by MOFs is also highly tunable, ${ }^{23}$ allowing for different biological responses to be targeted (e.g., vasodilation and anti-platelet aggregation). MOFs, therefore, offer the potential means to achieve local NO release from medical device surfaces at a range of biologically active concentrations, triggered by moisture, if they can be incorporated effectively into the devices.

Recently, we reported that NO can be successfully adsorbed and released by stand-alone polyurethane (PU) films containing up to $15 \mathrm{wt} \%$ hydrothermally produced CPO-27$\mathrm{Ni}^{38}$ Such films act as models for device coatings (PU being a commonly employed polymer in medical devices such as catheters). Despite finding that the PU matrix reduced the total quantity of $\mathrm{NO}$ adsorbed and released by the MOF, it was shown that the concentration of NO released from CPO-27$\mathrm{Ni}$-containing films elicited a bactericidal effect against Escherichia coli (E. coli) and Staphylococcus aureus (S. aureus). Importantly, cytotoxicity analysis of the MOF/PU films against human umbilical vein endothelial cells recorded low toxicity levels, and this toxicity was attributed to the released NO rather than the MOF or its components. The study proved the concept that NO-releasing MOFs may indeed offer a route to localized NO release from medical device surfaces, but it also highlighted the non-trivial nature of $\mathrm{NO}$ adsorption and release from MOFs embedded in a PU matrix. Further detailed analysis of the fundamental properties and behavior of MOF/ polymer films in the context of NO release is therefore warranted, not least to help establish underlying factors that influence performance.

In the present study, we seek to understand the fundamental contributing factors governing MOF/polymer compatibility in these composites and determine how they may relate to NO release efficiency. We aim to examine in detail the interactions of CPO-27-Ni with the model PU matrix, analyze the impact the MOF has on polymer mechanical properties (which are critical to end application), and explore any consequences of increasing the MOF loading beyond $15 \mathrm{wt} \%$. The MOF/ polymer interface has a crucial effect on the overall performance of the composite. In principle, the MOF can interact in three different scenarios with the supporting matrix. In the realm of mixed matrix membranes (MMMs) for gas separation, this is discussed as either "ideal", "sieve in cage", or "plugged sieve" MMM. For an MMM to be ideal, the MOF is highly compatible with the employed polymer and intermolecular forces between the MOF and the polymer result in a supportive composite. However, if the MOF/polymer interface is less supportive, the formation of (non-selective) macrovoids is observed, which directly leads to loss in performance (socalled sieve in cage). In the case of plugged sieve MMMs, poor performance results from full encapsulation of the MOF by the polymer and blockage of the MOF pores. Probing the MOF/ polymer interface proves challenging as it often involves (more than one) an advanced spectroscopic and/or microscopic technique. Consequently, only a few studies have reported the full characterization of the MOF/polymer interface; a summary of which can be found elsewhere. ${ }^{39-41}$ In this study, we employ for the first time a novel combination of force field molecular dynamics (MD) simulations, mechanical testing, advanced focused ion beam scanning electron microscopy (FIB-SEM), and water adsorption measurements. This detailed analysis is used to identify the optimum wt \% loading of MOF to achieve maximum NO release. As an illustration of the effectiveness of the optimal formulation, as found in this study, we also report antibacterial efficacy results against Pseudomonas aeruginosa to augment the suite of bacteria against which NO-releasing MOF/PU films have been tested. CPO-27-Ni was selected for the study as it has proved to offer high and efficient NO release and is thus an excellent model material. In contrast to our previous work, we use CPO-27-Ni MOFs obtained through two different synthesis paths: a hydrothermal route involving tetrahydrofuran (THF) co-solvent and a purely water-based route conducted at room temperature and ambient pressure. The latter is of interest as it carries higher commercial upscale potential.

\section{MATERIALS AND METHODS}

All chemicals used have been sourced from common suppliers without further purification (Table S1).

Synthesis and Characterization of CPO-27-Ni Powders. CPO-27-Ni was synthesized via two different methods, both previously reported in the literature: a hydrothermal THF/water-based synthesis (CPO-27-Ni-HT) ${ }^{30}$ and a waterbased, room-temperature synthesis (CPO-27-Ni-RT). ${ }^{42}$ Synthesis of CPO-27-Ni-HT powder: In a Teflon liner $(600 \mathrm{~mL})$, nickel acetate tetrahydrate $(18.67 \mathrm{~g}, 75.0 \mathrm{mmol})$ was dissolved in $\mathrm{H}_{2} \mathrm{O}(175 \mathrm{~mL})$. A solution of 2,5-dihydroxyterephthalic acid (dhtp) $(7.43 \mathrm{~g}, 37.5 \mathrm{mmol})$ in THF $(175 \mathrm{~mL})$ was then added and left to stir for $15 \mathrm{~min}$. Subsequently, the autoclave was sealed and reacted for 3 days at $110{ }^{\circ} \mathrm{C}$. A mustard-yellow powder was obtained with a yield of $92 \%$ (based on nickel). Synthesis of CPO-27-Ni-RT powder: nickel acetate tetrahydrate $(12.9 \mathrm{~g}, 50 \mathrm{mmol})$ was dissolved in water $(150 \mathrm{~mL}) .2,5$ dhtp ( $5 \mathrm{~g}, 25 \mathrm{mmol}$ ) was dissolved in $1 \mathrm{M}$ aqueous $\mathrm{NaOH}(25$ $\mathrm{mL}$ ) and added to the metal solution under stirring at room temperature. The reaction was stopped after $6 \mathrm{~h}$, and the resulting precipitate was extracted via filtration and washed with water. After drying in air, a mustard-yellow powder was obtained with a yield of $91 \%$ (based on nickel).

Powder X-ray diffraction (PXRD) analysis of large samples $(>1 \mathrm{~g})$ was carried out on a Panalytical Empyrean diffractometer in reflection mode. Smaller sample sizes were packed in capillaries and analyzed on a STOE STADIP diffractometer in transmission mode. Both diffractometers operate with $\mathrm{Cu} \mathrm{K} \alpha_{1}$ radiation monochromated with a curved $\mathrm{Ge}(111)$ crystal. Thermogravimetric analysis (TGA) was carried out on a Stanton Redcroft STA-780 simultaneous TG-DTA, in air. The samples were heated from 20 to $700{ }^{\circ} \mathrm{C}$ at a heating ramp rate of $5{ }^{\circ} \mathrm{C} / \mathrm{min}$. SEM analysis of the MOF powders was carried out as described below. Nitrogen isotherm data were collected on a Micromeritics ASAP 2020. MOF powders were activated in vacuo for $16 \mathrm{~h}$ at $150{ }^{\circ} \mathrm{C}$, at a heating rate of $10^{\circ} \mathrm{C} / \mathrm{min}$. Brunauer-Emmett-Teller (BET) surface area was determined according to the Rouquerol-BET theory ${ }^{43}$ using Micromeritics Active software (version 
4.03.04). Particle size distributions (PSDs) of as-made and dispersed MOFs were measured using a Malvern Mastersizer 2000 light scattering particle size analyzer, employing water as the dispersion media. Three measurements were taken under stirring $(1800 \mathrm{rpm})$ and a further three were recorded (under stirring) after a $3 \mathrm{~min}$ sonication cycle; each set of three measurements were averaged.

Preparation and Characterization of CPO-27-Ni Composites. A medical grade PU (chemical composition specified in Table S1 and Figure S9) was dissolved in THF. Prior to addition, the MOF was mechanically dispersed in THF. The MOF dispersion was then added to the polymer solution to obtain a certain wt \% of MOF calculated per gram of polymer. In this study, the range of composite materials prepared via doctor blade casting contained 5, 10, 20, and 40 wt $\%$ of MOF. SEM micrographs were acquired using a FEI Scios Dualbeam SEM, equipped with a FIB- $\mathrm{Ga}^{+}$column. Samples were placed on an aluminum stub with the aid of a carbon-based conductive adhesive. To improve conductivity, samples were brushed with a silver paste and sputter-coated with gold using a Quorum Q150R ES apparatus, operating at $10 \mathrm{~mA} / 30 \mathrm{~s}$. For imaging, the acceleration voltage ranged from 2 to $5 \mathrm{kV}$ at a working distance of $7 \mathrm{~mm}$ to ensure sensitive mapping of the surface. Additional microstructural analyses such as cross sections and 3D reconstructions were conducted using a FIB-SEM. To protect the sample from high voltages and currents, needed for FIB-milling, a thin platinum layer (20 $\times 2 \times 1 \mu \mathrm{m})$ was deposited via ion beam induced deposition on the region of interest. Subsequently, the FIB was employed to mill cross-section trenches $(20 \times 15 \times 7.5 \mu \mathrm{m})$ at $>1 \mathrm{nA}$ current; the obtained cross-section was polished at $<1 \mathrm{nA}$ current. The SEM column, oriented $52^{\circ}$ to the FIB, could then be used to image the microstructure revealed by the crosssection. 3D reconstructions were prepared by milling further trenches on either side of a cross section of the area of interest. Once isolated, the individual cross sections are compiled into a $3 \mathrm{D}$ reconstruction. Analysis and rendering of the reconstruction volume were conducted using the Thermo Scientific Avizo Software 9.3.0 kit.

Activation of MOF Composites, Nitric Oxide Loading, and Release Measurements. Each MOF composite was cut into $2 \times 2 \mathrm{~cm}$ samples, loaded into glass ampoules, and activated for $16 \mathrm{~h}$ at elevated temperatures, in vacuo $\left(80{ }^{\circ} \mathrm{C}\right.$, $10^{-4}$ mbar). After activation, the composites were bathed in a NO atmosphere ( 2 bar absolute pressure) for $1 \mathrm{~h}$. Two argon flushes (each extracted under vacuum) were applied to remove excess and physisorbed $\mathrm{NO}$, after which the ampoules were flame-sealed under dry argon to prevent premature release of chemisorbed NO. Kinetic NO release measurements were carried out within hours of loading the MOF composite samples with NO. These data were recorded using a Sievers NOA chemiluminescence analyzer as previously reported. ${ }^{38}$ The ampoules were snapped open, and the composites were placed directly into a sample chamber under a flow of $\mathrm{N}_{2}$ gas with a relative humidity $(\mathrm{RH})$ of $11 \%$. Data acquisition was stopped once the NO release concentration fell below $30 \mathrm{ppb}$, as these levels are near the detection limit of the analyzer. The $\mathrm{NO}$ analyzers record the amount of $\mathrm{NO}$ that is being released from the material in $\mathrm{ppm} / \mathrm{ppb}$, producing a concentration profile of $\mathrm{ppm} / \mathrm{ppb}$ versus time. By accumulating the values of this concentration profile, an integral (area under the curve) is obtained that indicates the total release in units of mmol of NO. These values are normalized to either the mass of the film or mass of the MOF within the film to allow direct comparison between samples. The resulting graphs therefore depict total NO release in mmol per gram of the film or MOF versus time. Each measurement was recorded in duplicate. For the purposes of calculating the mass of the MOF present in each film, it was assumed that the wt \% of the MOF within the $2 \times 2 \mathrm{~cm}$ sample was equal to that added to the polymer dispersion during film fabrication and equal for each replicate of given wt \%. The mass of the MOF present was therefore calculated from the mass of each film sample. It was also assumed that, by employing a $2 \times 2 \mathrm{~cm}$ sample, any potential variation in $\mathrm{NO}$ release between samples caused by any local (i.e. micron-scale) variation in MOF distribution would be rendered negligible.

Water Adsorption Isotherm Data. Water adsorption isotherms were recorded on a DVS Advantage (Surface Measurement Systems Ltd.). The composite ( $30 \mathrm{mg})$ was placed on a stainless-steel (UltraBalance high mass) pan microbalance (resolution $1 \mu \mathrm{g}$ ). Prior to the experiment, each as-synthesized sample was degassed for $4 \mathrm{~h}$ at $80{ }^{\circ} \mathrm{C}$ in a dry atmosphere in order to standardize each sample. The sample was then subjected to a series of relative humidities (0-95\% $\mathrm{RH}$ ) under automatic control. At each $\mathrm{RH}$, the sample mass was monitored, and a data point was recorded at equilibrium; that is, when the rate of change fell below an arbitrarily defined level of $0.001 \%$ per min and remained below that value for at least $10 \mathrm{~min}$.

Modelling of the CPO-27-Ni/PU Composite. The CPO$27-\mathrm{Ni} / \mathrm{PU}$ composite model was built by combining separate MOF surface and polymer models, following a methodology developed previously. ${ }^{44}$ First, the $\{101\}$ surface model of the CPO-27-Ni material was cut from the crystal structure and reconstructed so as to ensure dipole neutrality along the $z$-axis. The final slab, consisting of 10 "layers", was further geometryoptimized at the density functional theory (DFT) level using the Perdew-Burke-Ernzerhof functional ${ }^{45}$ and DFT-D3 dispersion corrections ${ }^{46}$ as implemented in the Quickstep module of the CP2K software. ${ }^{47}$ An atomistic model of PU was constructed in silico by means of a force-field-based MD strategy previously reported with the use of polymatic ${ }^{48}$ software. A representative model of this polymer was built by considering the isocyanate together with di(propylene glycol) as a single polyol. A scheme of the repeat unit containing the isocyanate and the polyol is provided in Figure S9. Both bonded and non-bonded energy contributions to the force field were considered. Van der Waals interactions were modelled by 12-6 Lennard-Jones ( $\mathrm{LJ}$ ) terms with parameters taken from the TraPPE-UA $^{49}$ force field and Coulombic contributions with charges extracted using the restrained electrostatic potential (RESP) method. ${ }^{50}$ GAFF was considered to describe the bonded energy terms. ${ }^{51}$ The resulting polydisperse polymer model contains chains with sizes ranging between 15 and 46 monomers totaling 148 monomers. The model was validated by computing the polymer density after equilibration, which was found to be $1.14 \mathrm{~g} / \mathrm{mL}$, in good agreement with the experimental value of $1.18 \mathrm{~g} / \mathrm{mL}$. The polymer model was then put into contact with the CPO-27-Ni $\{101\}$ surface model, the MOF surface being located in the middle of the box. All atoms of the MOF framework were maintained in their initial positions. The resulting MOF/composite system was equilibrated by a cycle of force field-based MD simulations in the $N V T$ and $N P_{\mathrm{n}} T$ ensembles, where $P_{\mathrm{n}}$ is the pressure normal to the MOF surface ( $z$-direction). A modified version of DLPOLY classic ${ }^{52}$ was used for the interface generation and 
production simulations. $\mathrm{MOF} /$ polymer interactions were modelled by the sum of 12-6 LJ terms and electrostatic terms. Crossed LJ parameters were determined by applying the Lorentz-Berthelot mixing rules. The LJ parameters of the CPO-27-Ni surface were taken from UFF $^{53}$ and DREIDING ${ }^{54}$ force fields for the inorganic nodes and organic linkers, respectively, while the charges were derived from the previous set of RESP charges calculated for the bulk crystal. ${ }^{55}$ To circumvent sampling issues, seven different initial composite configurations were optimized and further analyzed with production runs $10 \mathrm{~ns}$ long each. This procedure was used and validated by comparing with biased potential dynamics results in our previous work, where the methodology for building MOF/polymer interfaces was first developed. ${ }^{4}$ The properties of the composites discussed below are averaged over this whole set of configurations. All details of the computational methodology and parameters are provided in the Supporting Information.

Mechanical Testing. Uniaxial tensile tests were performed using a Hounsfield desktop universal testing machine equipped with a $250 \mathrm{~N}$ load cell. The gauge length and width of the film specimens were 10 and $5 \mathrm{~mm}$, respectively. The thickness of the films was typically $\sim 100 \mu \mathrm{m}$. Specimens of the 5 different MOF-loadings were tested $(0,5,10,20$, and $40 \mathrm{wt} \%)$, and a constant displacement rate of $10 \mathrm{~mm} / \mathrm{min}$ was applied. The raw data recorded were tensile force as a function of uniaxial extension, from which the nominal stress (=force/original cross-sectional area) and nominal strain (=extension/original gauge length) were calculated. Mechanical toughness of the film was characterized by evaluating its fracture energy $\left(\mathrm{J} / \mathrm{m}^{2}\right)$, obtained by integrating the area under the stress-extension curve. $^{56}$

Antibacterial Testing. Antibacterial activity of MOF composite films (typically $\sim 100 \mu \mathrm{m}$ thickness) was tested against $P$. aeruginosa ATCC 15442 . The composite films $(N=$ 6) were cut into $2 \times 2 \mathrm{~cm}$ samples and placed into individual wells of a 12 well plate. The single strain of $P$. aeruginosa was inoculated in $10 \mathrm{~mL}$ of tryptone soya broth (TSB) and incubated overnight at $37{ }^{\circ} \mathrm{C} / 125 \mathrm{rpm}$ in an orbital shaking incubator. The obtained Pseudomonas aeruginosa(P. aeruginosa) culture was adjusted with TSB to achieve a bacterial density of $\sim 1 \times 10^{6} \mathrm{CFU} / \mathrm{mL}$. The adjusted inoculum $(0.5 \mathrm{~mL})$ was directly pipetted onto the surface of the films, which were then incubated for 5 and $24 \mathrm{~h}$, respectively at $37^{\circ} \mathrm{C}$. After each time point, the films were transferred to Dey England neutralizing broth and vortexed briefly. Aliquots were then serial diluted with phosphate saline buffer and pipetted $(50 \mu \mathrm{L})$ onto tryptone soya agar in a Petri dish. The plates were incubated at $37{ }^{\circ} \mathrm{C}$ overnight after which the bacterial counts were enumerated; the results are expressed as bacterial density ( $\mathrm{CFU} / \mathrm{mL})$.

\section{RESULTS AND DISCUSSION}

Synthesis and Characterization of CPO-27-Ni Powders. MOFs are often made under relatively harsh conditions using organic solvents such as dimethylformamide or THF to improve crystallinity. For commercial upscaling, synthesis procedures employing less energetically intensive and timeconsuming conditions and more environmentally friendly solvents would be desirable. In this study, we use CPO-27Ni MOFs obtained through both approaches: a THF-based hydrothermal method (material referred to as CPO-27-NiHT) and a previously disclosed water-based, room temper- ature and highly scalable method (material referred to as CPO27-Ni-RT) to compare their respective interactions with PU. On comparing the PXRD patterns of both CPO-27-Ni-HT and CPO-27-Ni-RT with that of a pattern simulated from single crystal PXRD data for CPO-27-Ni, we can conclude that both synthesized materials are pure phase (Figure S1a). As reported previously, ${ }^{42}$ the MOF afforded from the hydrothermal synthesis displays narrower and sharper PXRD reflections and is thus of higher crystallinity. It is also evident from BET analysis (Figure S2) that the material prepared hydrothermally possesses a greater surface area; BET areas of 902 and $283 \mathrm{~m}^{2} / \mathrm{g}$ were recorded for CPO-27-Ni-HT and CPO-27-Ni-RT, respectively. For any industrial application, the stability of all materials throughout the entire product fabrication process is crucial. The stability of the synthesized MOFs toward mechanical dispersion and storage in THF (employed in this study to process the polymer films) and water (as the final application is in moist media) was therefore also tested. Dispersions of CPO-27-Ni-RT in THF and water were stored for six months. Aliquots were removed after $12 \mathrm{~h}$ and 6 months, dried in air, and analyzed by PXRD in transmission mode. Each of the collected PXRD patterns (Figure S1a) is consistent with that of the starting material, suggesting that CPO-27-Ni-RT is stable in water and THF even after an ageing period of 6 months.

To unlock the functionality of $\mathrm{CPO}-27-\mathrm{Ni}$, solvent molecules that are bound to the metal centers in the asmade material must be removed. This process, which is often referred to as activation, is typically conducted through heat treatment and therefore the thermal stability of the MOF must be considered. Figure S1b shows the thermogravimetric profiles for both MOFs, which display almost identical thermal behavior that is consistent with previous reports. ${ }^{42} \mathrm{~A}$ significant mass loss of $32 \%$ is observed in the range of $20-$ $150{ }^{\circ} \mathrm{C}$. This is frequently observed for this type of MOF and is commonly attributed to the loss of water and/or solvent employed in the synthesis. ${ }^{23,30,57}$ Both materials are stable up to $270{ }^{\circ} \mathrm{C}$ (beyond which decomposition occurs), giving a wide thermal processing window, where CUSs are available and the framework remains intact. Figure S1c,d depicts the representative SEM micrographs of the as-made MOF powders. The micrographs indicate that both materials comprise agglomerated microcrystalline powder. PSDs measured in water suggest $d(0.5)$ values of 13.10 and $9.45 \mu \mathrm{m}$ for CPO-27-Ni-HT and CPO-27-Ni-RT, respectively, with a broader overall distribution for CPO-27-Ni-RT (Table S2).

Preparation and Characterization of CPO-27-Ni Composites. The connectivity of the components employed to prepare functional composite materials such as MMMs has proved to be highly important to overall performance. ${ }^{39}$ Good dispersion and limited agglomeration of the MOF particles in the polymer matrix are important in controlling the effectiveness of the final composite, and as such good compatibility between the MOF and the polymer is desirable. This is the case when smaller, uniform particles and morphologies are used for the composite material. ${ }^{41}$ Of course, altering the architecture and composition of the MOF itself to tune the interaction between the MOF and guest adsorbate can also be a powerful means to control the properties of the composite. For the purposes of this study, however, we focus on the interactions between the MOF and polymer. 
Prior to preparing the composite materials, the MOF powders were mechanically dispersed in THF to achieve narrower and more uniform PSDs compared to those offered by the as-made material. PSD analysis (Table S2 and Figure S3) indicates that the mechanical dispersion process helps to generate smaller particle sizes. While the distribution obtained from CPO-27-Ni-RT is narrow and unimodal (centered at $\sim 2.5 \mu \mathrm{m}$ ), the distribution of CPO-27-Ni-HT is bimodal with populations centered at $\sim 0.12$ and $1.5 \mu \mathrm{m}$. The growth of a particle population below $1 \mu \mathrm{m}$ in both samples upon application of sonication inside the particle size analyzer chamber suggests that the distributions of particles obtained by mechanical dispersion and recorded without sonication are still agglomerates of primary particles, albeit they are much smaller than the agglomerates of the as-synthesized materials. It is believed from SEM analysis that the true primary particles of both samples are in the nanometer range. Particles of such dimensions are notoriously difficult to sustain in dispersion without agglomerating, and it is evident that the dispersions employed to prepare the films comprise agglomerates. It should be noted that only sheer forces (from mechanical stirring) were employed during the preparation of the films; therefore, the dispersions introduced to the films were defined as "dispersed" in Table S2.

Composite materials comprising 5, 10, 20, and 40 wt \% of CPO-27-Ni-HT and CPO-27-Ni-RT were prepared using a medical-grade PU as outlined above. The composite materials, cast as thin films, act as a proxy for a thin catheter coating. The films are self-supporting, pliable, and soft to the touch and appear to have a smooth surface to the eye. ${ }^{38}$

SEM micrographs were recorded to investigate the surface of the CPO-27-Ni-HT and CPO-27-Ni-RT composite films containing $5 \mathrm{wt} \%$ (Figure S4), $10 \mathrm{wt} \%$ (Figure 1a,b), $20 \mathrm{wt} \%$
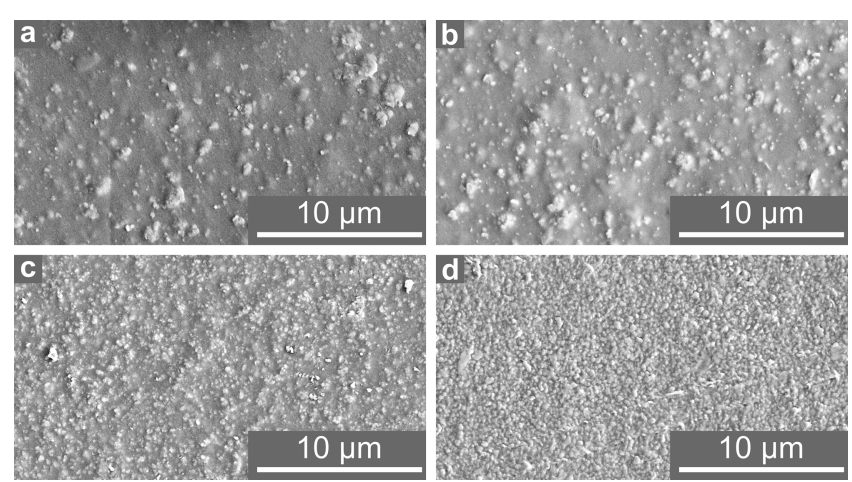

Figure 1. SEM micrographs of the surface of MOF composite films. (a) CPO-27-Ni-HT-10 wt \% MOF, (b) CPO-27-Ni-RT-10 wt \% MOF, (c) CPO-27-Ni-HT-40 wt \% MOF, and (d) CPO-27-NiRT-40 wt \% MOF.

(Figure S5), and 40 wt \% (Figure 1c,d). As anticipated from the PSD analysis, the micrographs reveal that the MOF is present as agglomerates. Importantly, these agglomerates are distributed across the length and breadth of the films, fully embedded by the matrix with no particles exposed. They are also of dimensions akin to those recorded above following mechanical dispersion, which indicates that no further significant agglomeration has occurred as a result of being incorporated into the PU. Composite materials with 5 and 10 wt \% MOF loading appear to have areas of "empty" polymer, while those containing higher MOF loadings exhibit a more densely packed MOF, as would be expected. PXRD analyses of the full range of MOF films showed that the MOF's crystallinity is retained throughout the preparation of the MOF polymer composites (Figure S6), indeed with increasing wt \% loading, there is an increase in intensity of the peaks attributable to $\mathrm{CPO}-27-\mathrm{Ni}$.

The highly efficient and reversible nature of $\mathrm{NO}$ storage and release exhibited by powder-form CPO-27-Ni has been widely reported. ${ }^{11,42}$ However, to safely realize the potential of these materials as NO-releasing agents within medical devices, functionality must be achieved not in the powder form but when formulated and shaped into polymeric articles. ${ }^{38,58,59}$ This creates an additional challenge in that the composite formulation and activation/NO loading conditions must be compatible. Traditionally, the activation of CPO-27-Ni is conducted at temperatures above $100{ }^{\circ} \mathrm{C}$, as indicated by the TGA profile. However, as the PU employed in producing the films is thermoplastic with a softening point of $85{ }^{\circ} \mathrm{C}$, such activation temperatures could not be used. Instead, activation was carried out at $80{ }^{\circ} \mathrm{C}$ in vacuo. PU films are clear and colorless but take the color of the employed MOF when prepared as MOF composite films (yellow-brown in the case of CPO-27-Ni, Figure S7a). It has been observed previously that the MOF powder changes color to a darker brown/black upon adsorption of NO. ${ }^{42}$ This change in color is a result of the coordination of NO to the CUSs created upon activation. ${ }^{60} \mathrm{~A}$ similar color change is observed here for the composite materials and serves as visual confirmation that the activation and NO-loading was successful. As expected, the intensity of this visual effect varies with the MOF loading level (Figure $\mathrm{S} 7 \mathrm{~b})$.

NO release performance of the full range of activated and NO-loaded composite films was recorded under $11 \% \mathrm{RH}$ and is illustrated in Figure 2. Data are plotted per gram of film and per gram of MOF within the films. Exact NO release levels and the duration of release for each composite can be found in Table S3. NO release was recorded using a gas-phase system as outlined earlier-that is, a humid atmosphere triggers the release of NO, which is carried to the analyzer by flowing nitrogen. Although this method employs a lower $\mathrm{RH}$ than would be experienced by the materials under physiological conditions, it allows for all released NO to be detected. Methods employing $100 \% \mathrm{RH}$ (i.e. submersion) may underestimate the quantity of $\mathrm{NO}$ released due to its low water solubility. Although the employed $\mathrm{RH}$ is lower than that of physiological conditions, employing a low $\mathrm{RH}$ allows for release to be triggered in a more controlled manner and provides the opportunity to identify nuances in release profiles that may be lost due to faster kinetics under higher humidity. Such control is preferable in the present study which seeks to analyze fundamental relationships between film properties.

The shapes of the release profiles of the composites are consistent with those of $\mathrm{CPO}-27-\mathrm{Ni}$ powder (reported elsewhere), ${ }^{38,42}$ albeit the release kinetics are retarded, and concentrations attenuated by the polymer matrix. This is in agreement with our previous findings and emphasizes the importance of the polymer matrix in relation to achieving the desired performance; in order for NO to be released from the films, water must permeate the polymer matrix before reaching the NO-loaded MOF. Although the temperature of activation $\left(80{ }^{\circ} \mathrm{C}\right)$ was much lower than those traditionally employed, and lower than what might be expected to be necessary in order to activate the MOF, the release of $\mathrm{NO}$ by the films 

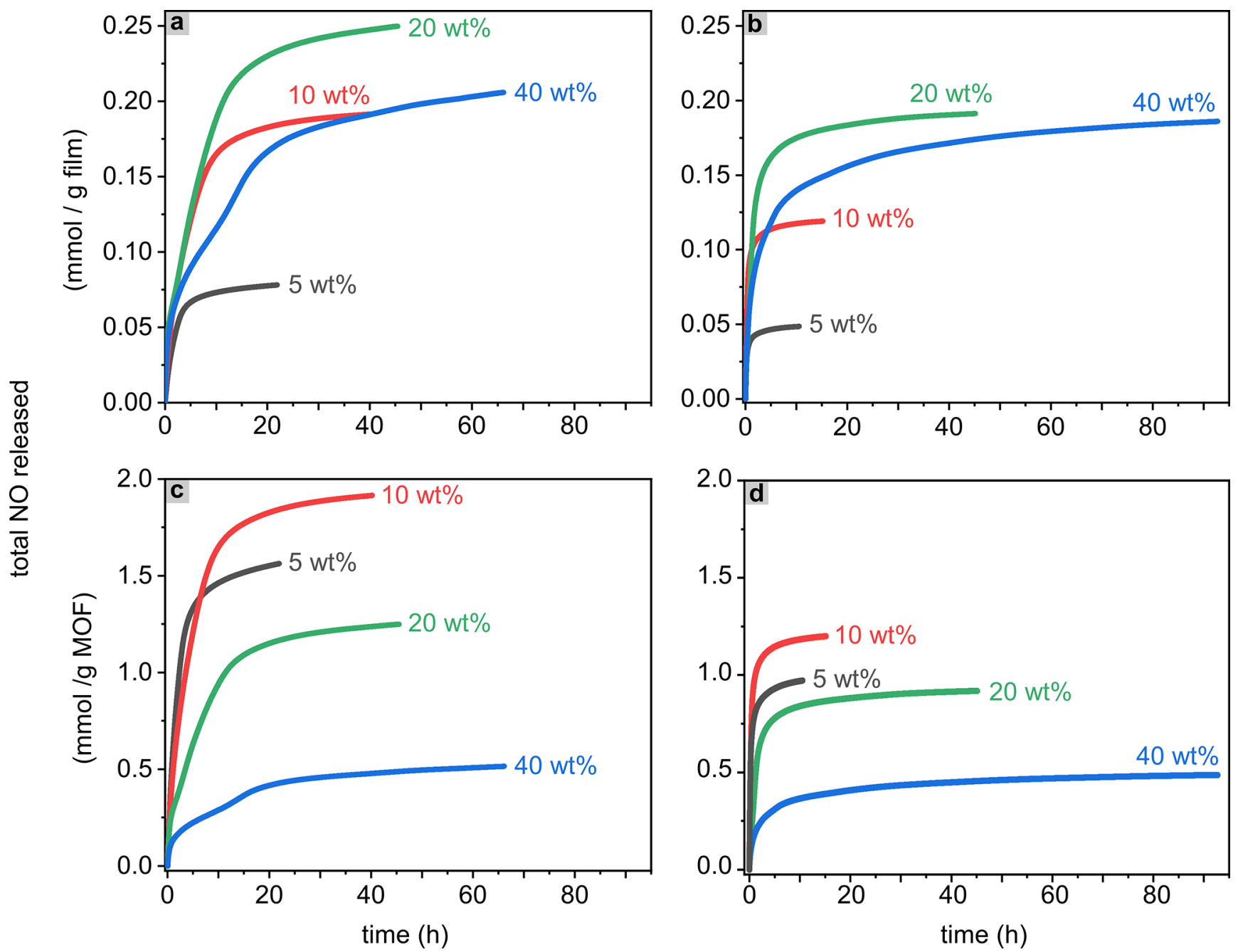

Figure 2. Kinetic NO release profiles expressed as total number of mmol of NO released per gram of film: (a) CPO-27-Ni-HT and (b) CPO-27Ni-RT and per gram of MOF: (c) CPO-27-Ni-HT and (d) CPO-27-Ni-RT composite films with varying MOF loading levels. Measurements were stopped once the concentration of NO release fell below $30 \mathrm{ppb}$.

confirms that the MOF therein had indeed been successfully activated at the lower temperature. This was a surprising result and indicates that activation of these materials warrants further investigation. Generally, composite materials containing CPO27-Ni-HT release slightly more NO compared to those containing CPO-27-Ni-RT, though it should be noted that the concentrations of NO released from all films are sufficient to elicit biological responses. This contrasting behavior may be attributed to the higher crystallinity and surface area of the hydrothermal material. It is possible that the more crystalline the material, the more accessible are the metal sites in the unit cell and the more efficient is the storage and release cycle of $\mathrm{NO}$ in the composite material (see Figures S1a and S2). It is interesting to note, however, that the difference between the quantities of $\mathrm{NO}$ released by the materials is not commensurate with the difference between their BET surface areas. Poor correlation between BET surface area and NO adsorption/release performance has been noted previously. ${ }^{42}$ At present, we propose this phenomenon is due to the differences between the conditions employed and mechanisms involved in the adsorption of nitrogen versus the adsorption and release of $\mathrm{NO}$, though this does require further investigation. A further aspect, i.e., the subject of ongoing investigation is the influence of film thickness on NO release.
Indeed, other works on MOF membranes for gas separation have found that the composite thickness is an important factor that directly influences permeability of the final composite. ${ }^{61,62}$ In our case, it may be assumed that penetrating water will contact MOF particles close to the surface of the films first. Therefore, the release characteristics of MOF particles close to the film surface may be quite different to those of particles in the center of the film. These aspects will be discussed in a later report.

By considering the release data in units of total mmol $\mathrm{NO}$ released per gram of film, the $\mathrm{NO}$ release of the final composites can be compared, see Figure 2a,b. Intuitively, and as we have reported previously for films containing $1,5,10$, and 15 wt $\% \mathrm{MOF}^{38}$ we would expect that higher MOF loading levels in the composites would translate to higher $\mathrm{NO}$ release concentrations. Indeed, this trend is observed up to a MOF loading of $20 \mathrm{wt} \%$. However, the data show that the higher loading of $40 \mathrm{wt} \%$ releases much less NO than would be expected and releases less NO per gram of the film than films containing $20 \mathrm{wt} \%$. This is true for composites containing both types of CPO-27-Ni HT and RT. This is the first time NO release has been characterized in films containing greater than 15 wt \% CPO-27-Ni, and the results 
a

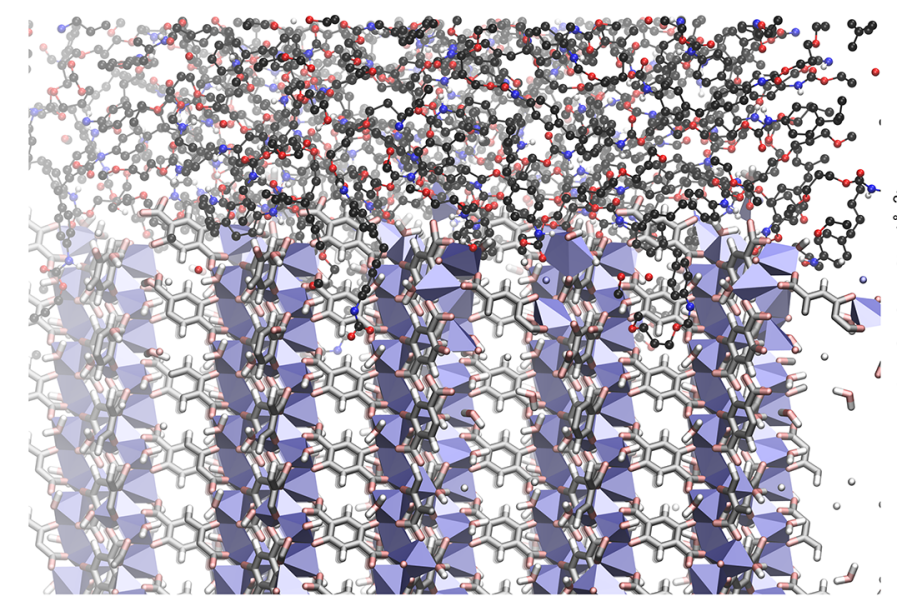

b
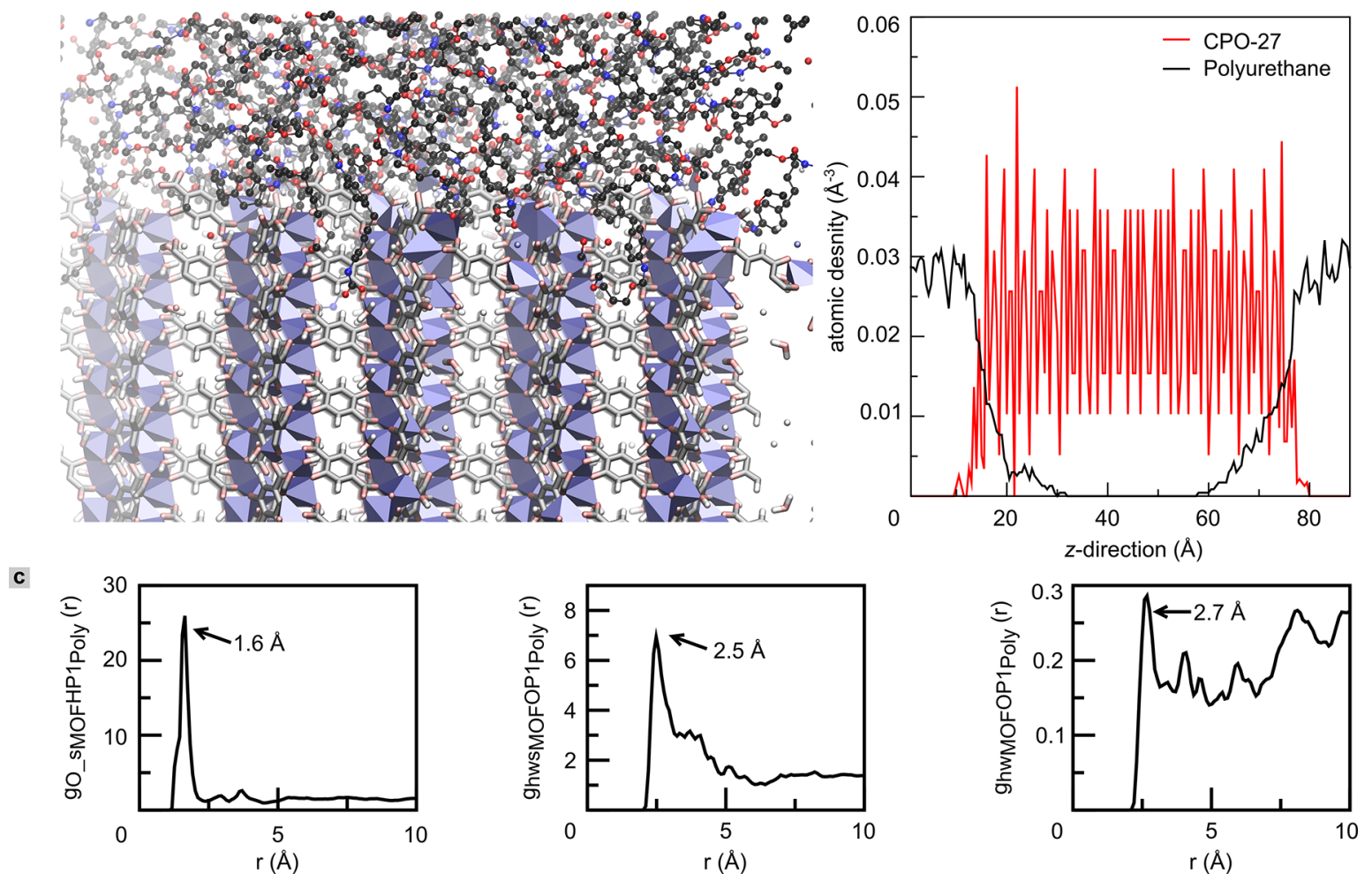

Figure 3. (a) Representative snapshot of the modelled CPO-27-Ni/PU interface. The MOF atoms are represented by a light-tone stick/polyhedra model, while an opaque ball and stick model represents the polymer. Color code: C (black), O (red), $\mathrm{H}$ (white), N (blue), and Ni (blue polyhedra). (b) Atomic density for PU (black) and CPO-27-Ni (red) as a function of the direction normal to the MOF surface. (c) Relevant average radial distribution functions between the surface $\mathrm{O}_{\mathrm{CPO}-27-\mathrm{Ni}}$ and the amide $\mathrm{H}_{\mathrm{PU}}$ (left panel) and $\mathrm{H}_{\mathrm{CPO}-27}$ from surface water molecules and the amide $\mathrm{O}_{\mathrm{PU}}$ (middle panel). In the right panel, the most relevant interaction for the bulk is shown: $\mathrm{H}_{\mathrm{CPO}-27-\mathrm{Ni}}$ from inner water molecules with $\mathrm{O}_{\mathrm{PU}}$ (for force field atom types see Supporting Information). The average distances between the sites are highlighted in each case.

suggest the possible existence of an optimum formulation for maximizing the total concentration of $\mathrm{NO}$ released.

To gain insight into the NO release efficiency of the MOF inside the polymer matrix, the NO release data are further analyzed per gram of the MOF inside the final composite (Figure 2c,d). Our previous analysis indicated that PU films containing CPO-27-Ni-HT adsorb the same quantity of NO per gram of the MOF regardless of wt \% loading (as would be expected). ${ }^{38}$ Intuitively, there is no reason to predict that the MOF's ability to release its adsorbed payload should vary with wt \% loading either. However, the values reported in Figure $2 \mathrm{c}, \mathrm{d}$ suggest that this is not the case. Using this interpretation of the data, it is evident that the NO release efficiency of films containing $10 \mathrm{wt} \% \mathrm{MOF}$ is higher than that of films containing 5, 20, and 40 wt \% MOF. Although we had previously reported that the efficiency of release of films containing $10 \mathrm{wt} \%$ was higher than that of films containing 5 wt $\%,{ }^{38}$ it is surprising to observe here the dramatic reduction in release efficiency at MOF loadings of 20 and $40 \mathrm{wt} \%$. It seems, therefore, that an optimal MOF loading for maximum NO release concentration is reached at around $10 \mathrm{wt} \%$ (under the conditions used in this study) and that higher MOF loadings result in reduced release efficiency. This would appear to be the case regardless of which powder CPO-27-Ni material is used; it must be realized, however, that although high NO concentrations are required for antibacterial applications, lower concentrations are more appropriate to trigger other responses such as vasodilation. ${ }^{5}$ Therefore, the true "optimum" wt \% is rather dependent on the targeted end application.

Comparing the quantity of NO stored to the quantities released per gram of MOF from the films analyzed here indicates that not all stored $\mathrm{NO}$ is released over the time period studied. This is reflected by the film retaining a brown coloration, which is indicative of residual NO bound to CUSs within the MOF. This suggests that NO release could continue at a low level (below $30 \mathrm{ppb}$ ) for a further significant length of time.

It is observed that the profiles for the $5 \mathrm{wt} \%$ composites show a fast burst release of NO for a short duration. On increasing the MOF loading level, the duration of release is significantly prolonged, indeed composite films containing 20 and $40 \mathrm{wt} \%$ can sustainably release at least $30 \mathrm{ppb}$ of $\mathrm{NO}$ over many days. These concentrations of NO are deemed to be biologically active. These data indicate that the amount of total $\mathrm{NO}$ and the duration (i.e., kinetics) of its release can be tuned by controlling the loading content of active material inside the supporting matrix.

The phenomenon of optimal loading inside a polymeric matrix is often observed in MMMs for gas separation, ${ }^{41,63,64}$ as agglomeration at high loadings and how the filler is embedded in the polymeric matrix have an impact on the permeability and diffusivity of the MMM. ${ }^{58,59,65}$ Furthermore, good compatibility between the inorganic filler and polymeric matrix is crucial to optimize the performance of composites. Therefore, to investigate the possible reasons for the observed 
relationship between NO release and MOF loading, we have conducted an investigation into the interaction between the MOF and polymer, the distribution and agglomeration of the MOF within the polymer, and how the MOF influences the mechanical and water adsorption properties of the composite film.

MD Simulations on the CPO-27-Ni/PU Composite. $\mathrm{MD}$ simulations were performed in order to gain insight into the MOF/polymer compatibility at the molecular scale. A typical simulation snapshot featuring the interface between $\mathrm{CPO}-27-\mathrm{Ni}$ and $\mathrm{PU}$ within the composite was analyzed (Figure 3a). In the simulation, PU is found to adapt its structure to the morphology of the MOF surface, and no interfacial voids could be detected. In addition, the simulation suggests that the polymer penetrates the pores of the MOF at the MOF surface. This qualitative analysis suggests that CPO27-Ni exhibits good compatibility with the chosen PU. A more detailed picture may be obtained from the atomic density of CPO-27-Ni and PU at the interface along the direction that is normal to the MOF surface (in this case, the $z$-direction of the simulation box) (Figure 3b). The MOF's density centered in the middle of the simulation box, depicted in red, exhibits evenly spaced peaks due to the regularity of its structure. The density profile for PU (black line) oscillates around a constant value closer to the endpoints of the simulation box and decays when the MOF density becomes non-zero. Interestingly, there is a region in $z$-space where both densities are non-zero, which represents the overlap/interpenetration of the two components at the interface. This region extends to a length of $14 \pm 4 \AA$ averaged over the seven independent MD runs, with the error set to the standard deviation of the data. The extent of MOF/ polymer overlap is significantly greater than that found for all other MOF/polymer composites modelled at an ambient temperature in the past, such as poly(vinyl alcohol)/ HKUST-1, ${ }^{59}$ poly(ethylene glycol), and poly(vinylidene fluoride)/UiO- $66^{65}(11.5,5$ and $6 \AA$ respectively). These findings confirm the excellent compatibility between the two phases. The high degree of MOF/polymer overlap is a signature of the presence of favorable interactions between the two components. Analysis of radial distribution functions (RDFs) can shed light on these interactions. Figure $3 \mathrm{c}$ shows the RDFs for the most relevant of these pair interactions. Strong $\mathrm{H}$-bonds between the oxygen atoms $\mathrm{O}_{\mathrm{CPO}-27-\mathrm{Ni}}$ (atom type O_s) at the MOF surface and the hydrogen atoms of the amide group of $\mathrm{PU} \mathrm{H}_{\mathrm{PU}}$ (atom type $\mathrm{HP1}$, see Figure $3 \mathrm{c}$ left panel) is observed, with a characteristic short separating distance of $1.6 \AA$ and a high intensity peak. The hydrogen atoms of the water molecules at the MOF surface $\mathrm{H}_{\mathrm{CPO}-27-\mathrm{Ni}}$ (atom type hws) also interact with the oxygen-atom of the amide group $\mathrm{O}_{\mathrm{PU}}$ (atom type $\mathrm{OP} 1$, see Figure $3 \mathrm{c}$ middle panel) associated with a characteristic separating distance of $2.5 \AA$. Since the polymer penetrates the porosity of the MOF, there are additional interactions between the oxygen atoms of the amide group $\mathrm{O}_{\mathrm{PU}}$ and the $\mathrm{H}_{\mathrm{CPO}-27-\mathrm{Ni}}$ that correspond to the coordinated water molecules at the inner pore wall (atom type hw, see Figure $3 \mathrm{c}$ right panel), with a characteristic separating distance of $2.7 \AA$. These simulations reveal that PU adapts its conformation to the morphology of CPO-27-Ni and participates in strong $\mathrm{H}$-bonding interactions with atoms at both the MOF surface and inner pore wall. Taken together, these properties result in the two components being highly compatible in forming composites. While compatibility of all components is a crucial condition for an effective composite material with desirable mechanical properties, the remarkably high compatibility of our chosen polymer with the structure of CPO-27-Ni could lead to a partial blockage of the pores and metal centers. ${ }^{65}$ This may be a contributing factor to the lower NO release values recorded for the films, when compared to the MOF powders (Table S3).

Mechanical Behavior. To experimentally confirm the good compatibility between CPO-27-Ni and PU predicted computationally, uniaxial tensile tests were carried out on a set of CPO-27-Ni-HT composite films containing 0, 5, 10, 20, and 40 wt \% MOF loading levels. Tensile strength (TS) is the maximum stress that the film can endure in the stress-strain curve, while ductility is given by the maximum strain (\%) of the stretched-out film at the point of failure. In this analysis, small strips of the composite materials were mechanically elongated to failure point under a uniaxial load; the mechanical behavior of the films is depicted in Figure 4.

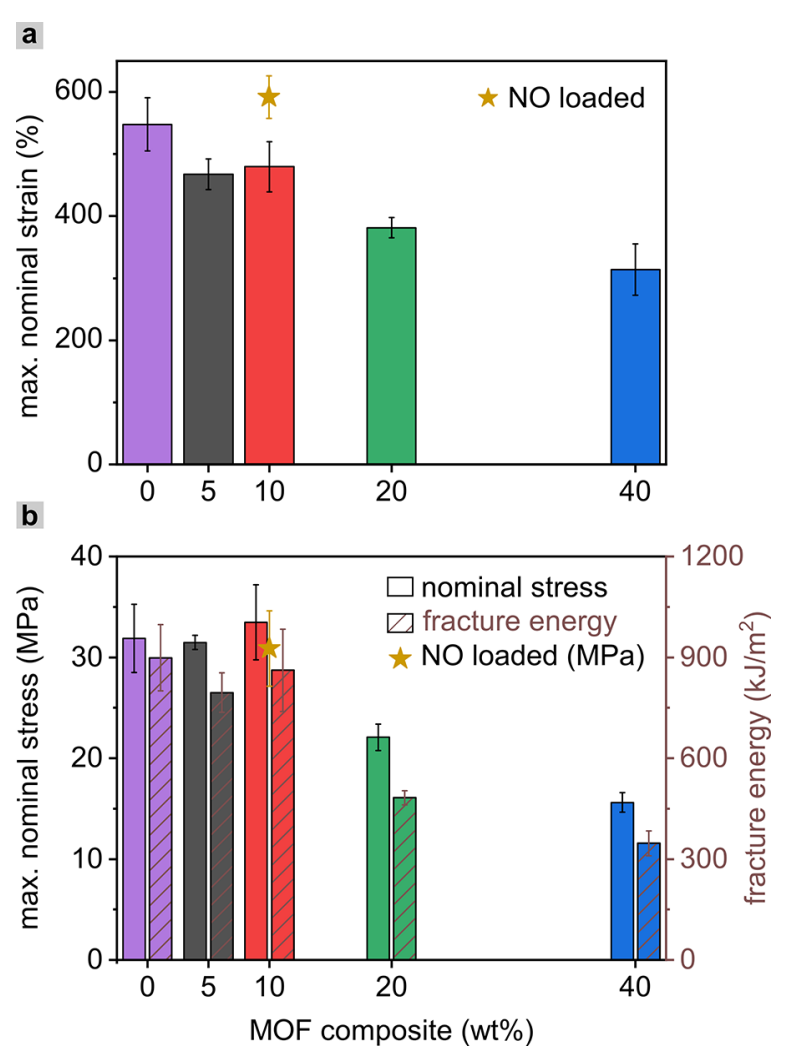

Figure 4. Mechanical testing results conducted on blank PU film (purple) and CPO-27-Ni-HT composite films containing 5 wt \% (black), 10 wt \% (red), 20 wt \% (green), 40 wt \% (blue), and NOloaded 10 wt \% (mustard star) MOF loading levels. (a) Ductility and (b) Tensile strength (TS) (left axis) and toughness (right axis).

The blank polymer film and composites of lower MOF content $(0-10$ wt \%) display a remarkable ductility with a high strain tolerance in the range of $\sim 480-600 \%$ (due to the rubbery behavior of PU). However, with higher MOF loadings (20 and $40 \mathrm{wt} \%$ ), the composites do become more brittle as evidenced by the reduction in strain tolerance (Figure $4 a$ ). Interestingly, the TS (Figure $4 \mathrm{~b}$ ) of the as-made composites remains high $(\sim 30 \mathrm{MPa})$ up to a threshold of $10 \mathrm{wt} \%$, beyond which the TS decreases significantly for 20 and 40 wt \% composites. For the $10 \mathrm{wt} \% \mathrm{MOF}$ composite, this results in a high fracture energy of $\sim 900 \mathrm{~kJ} / \mathrm{m}^{2}$ indicative of high 
mechanical toughness. The $10 \mathrm{wt} \%$ composite was also tested after an activation/NO-loading/release cycle had taken place. While the changes in TS are negligible, the ductility of the material slightly increased to $600 \%$, making the NO-loaded composite slightly more elastic. The maximum in fracture energy (at $10 \mathrm{wt} \%$ MOF loaded composite) occurs at the same MOF loading that releases the highest total NO concentration (per gram of MOF), and both NO release and mechanical toughness follow the same trend with respect to MOF loading. These findings point to a possible correlation between NO release performance and mechanical properties, governed by MOF wt \%. Overall, the excellent mechanical endurance of the composite films clearly confirms the prediction that the MOF/polymer compatibility is very good.

FIB-SEM Analysis. To further analyze $\mathrm{MOF} /$ polymer compatibility at the microscopic level and to investigate the morphology and three-dimensional distribution of MOFs within the films, FIB-SEM-assisted cross-sections were performed for the first time on the composite films.

The micrographs in Figure 5 depict the obtained crosssections of composites prepared using both CPO-27-Ni-HT

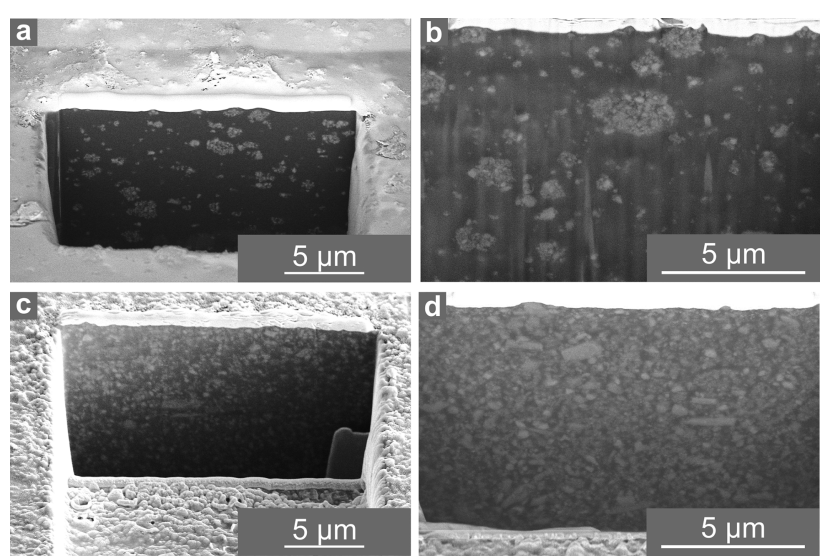

Figure 5. SEM micrographs of cross-sectional areas of CPO-27-Ni composite films. (a) CPO-27-Ni-HT-10 wt \% MOF, (b) CPO-27Ni-RT -10 wt \% MOF, (c) CPO-27-Ni-HT-40 wt \% MOF, and (d) CPO-27-Ni-RT-40 wt \% MOF.

and CPO-27-Ni-RT at 10 and 40 wt \%. Cross-sections of composites containing 5 and $20 \mathrm{wt} \%$ MOF are provided in Figures S4 and S5, respectively. In each case, MOF agglomerates are observed to be distributed throughout the entire depth of the film. Overall, a fairly similar agglomerate size is observed for both HT (Figure 5a,c) and RT (Figure $5 \mathrm{~b}, \mathrm{~d})$ MOFs with a range of $0.5-2.0 \mu \mathrm{m}$ for CPO-27-Ni-HT and $0.5-3.5 \mu \mathrm{m}$ for CPO-27-Ni-RT, respectively. Comparing the cross-sections of films containing 10 wt \% with those of films containing $40 \mathrm{wt} \%$, it becomes apparent that the polymer matrix is occupied by the MOF to a higher extent at higher MOF loadings, as might be expected. To visualize the distribution of the particles within the polymer, 3Dreconstruction experiments were carried out using the CPO27-Ni-HT-10 wt \% and CPO-27-Ni-HT-40 wt \% composite films. Figure 6 depicts the rendered volume that MOF particles occupy in each film.

As illustrated in Figure 6a, MOF particles are present as discrete agglomerates in the film containing $10 \mathrm{wt} \% \mathrm{MOF}$. Each new color represents an individual, discrete agglomerate. In contrast, in the case of higher $40 \mathrm{wt} \%$ loading, a much
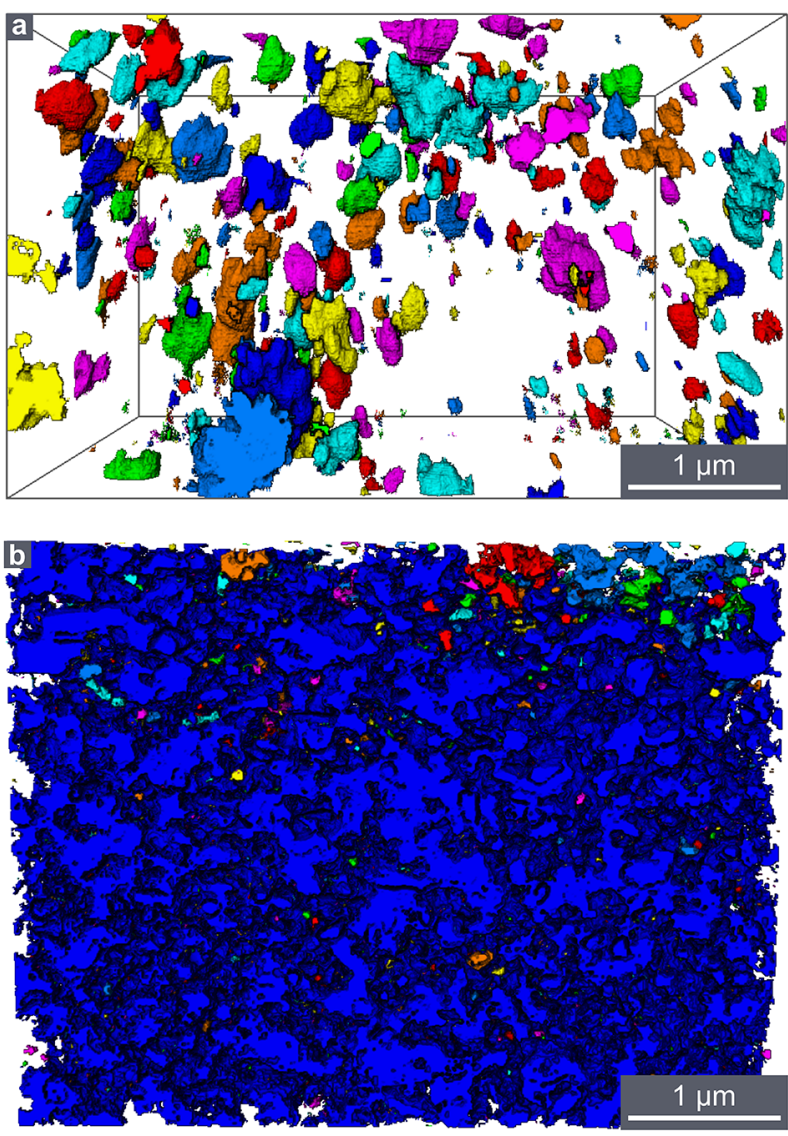

Figure 6. Rendered volume of $3 \mathrm{D}$ reconstruction experiment of CPO-27-Ni-HT composite films. (a) CPO-27-Ni-HT-10 wt \% and (b) CPO-27-Ni-HT-40 wt \% MOF. Cuboids with the dimensions $7.52 \times 3.47 \times 4.72 \mu \mathrm{m}$ and $8.24 \times 6.33 \times 4.44 \mu \mathrm{m}$ were analyzed for the 10 and $40 \mathrm{wt} \%$ films, respectively.

denser network of MOF particles with high connectivity and very few discrete particles is observed (note the monotone render in Figure $6 \mathrm{~b}$ highlighting widespread connectivity between agglomerates). The second phase (polymer) was omitted in both reconstructed volumes to allow for a more accurate calculation of the volume occupied by the MOF. The MOF within the 40 wt \% composite occupies $30.5 \%$ of the overall volume, whereas the MOF within the composite of 10 wt $\%$ occupies only $3.5 \%$. These analyses reveal for the first time that as the wt \% of the MOF in the films increases, MOF particles form a connected network within the polymer. This phenomenon is also described by the mathematical theory of a percolation threshold, which defines a maximum loading of the inorganic filler, above which a highly connected main component is formed within the composite system. This has previously been discussed in the realm of MMMs for gas separation. ${ }^{63}$ In our study, this higher connectivity and denser packing at higher loading could lead to overall fewer accessible MOF centres $^{66}$ and more complex gas transport behavior within the film. Both of these factors could contribute to the reduction in NO release concentration and may also be a contributing factor in the observed reduction in the ductility of these samples.

Water Adsorption Isotherms. To further probe the theory of more tortuous guest transport in films containing higher MOF wt \%, water adsorption analysis was also 
conducted on the samples. Water was selected because of its direct relevance to the mechanism of NO release; that is, an efficient NO storage and release cycle is achieved when the bound NO is replaced by water and all of the released NO is driven out of the material. ${ }^{11}$ However, the main purpose of the experiment was to provide evidence of guest transport characteristics.

Figure 7 illustrates water adsorption isotherms for films containing CPO-27-Ni-RT. The water uptake for the blank

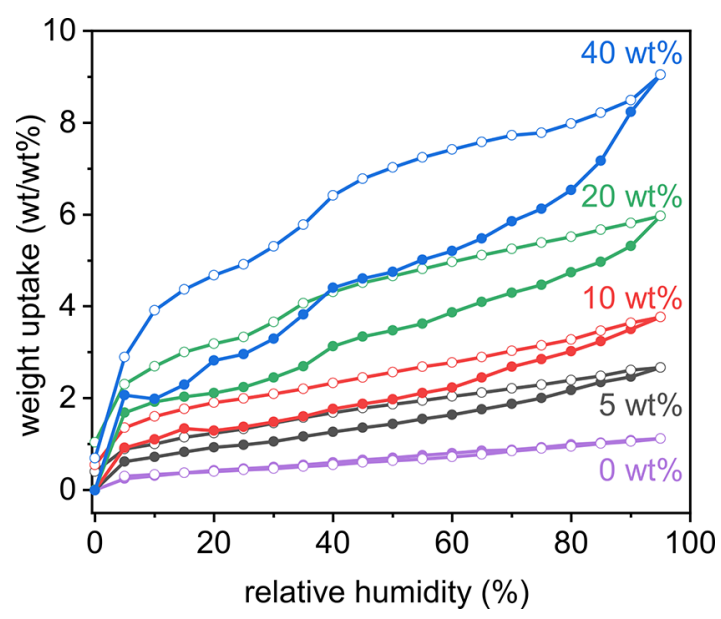

Figure 7. Water adsorption isotherms for blank PU and CPO-27-NiRT composite films containing 5, 10, 20, and $40 \mathrm{wt} \%$ MOF loading levels. Closed symbols refer to adsorption and open symbols to desorption.

polymer film is minimal; only $1.12 \mathrm{wt} / \mathrm{wt} \%$ uptake was recorded, indicating that this polymer is somewhat hydrophobic. However, with increasing MOF loading in the composite, the total uptake of water increases. This is not surprising as CPO-27-Ni-RT powder itself adsorbs $41.15 \mathrm{wt} /$ wt \% of water (Figure S8). The data indicate that, as reported previously for NO adsorption, ${ }^{38}$ incorporating the MOF into a polymeric matrix also attenuates its water adsorption capacity. This is likely caused by the polymer blocking access to MOF porosity.

For the isotherms of composites containing 5 and 10 wt \% MOF, a continuous uptake of water is observed. However, the isotherms of the 20 and $40 \mathrm{wt} \%$ composites exhibit a step-like profile where a sharp water uptake at low $\mathrm{RH}$ is followed by a small plateau in the isotherm before a second adsorption step at $\sim 40 \% \mathrm{RH}$. This behavior is most pronounced in the composite film with $40 \mathrm{wt} \% \mathrm{MOF}$ loading and is suggestive of staggered pore filling. The change in pore filling behavior at higher wt \% loadings coincides with changes in MOF packing discussed above, which is also reflected by the presence of a hysteresis gap between adsorption and desorption in these water isotherms (exact values are listed in Table S4). Although the exact reason for this remains unclear, it could indeed be consistent with more complex guest transport through these composites of high MOF content, but any definitive conclusion about guest transport derived from these data must be made with caution.

In view of the relationship between $\mathrm{NO}$ release and water adsorption, it is tempting to draw comparisons between these data sets. While this is possible to an extent, the following considerations are necessary, not least due to the fundamental differences in the conditions and methodologies of each technique. First, it must be noted that NO release data reported above were recorded at $11 \% \mathrm{RH}$, therefore corresponding water adsorption values must be compared under this same level of RH. Second, water adsorption isotherm data points are recorded under equilibrium conditions, and the isotherms do not directly yield information regarding the kinetics of adsorption [although we may infer some kinetic information from the total duration of each water adsorption experiment (Table S4)]. Third, the data do not indicate to which location the water has adsorbed within the composite material (each adsorbed water molecule may not necessarily exchange for NO at a CUS site). Finally, any comparison to $\mathrm{NO}$ release data must be made to data reported per gram of film (i.e. Figure $2 b$ ).

At $11 \% \mathrm{RH}$, the water uptake does not scale significantly with rising MOF content in the composites. Indeed, increasing the MOF content from 5 to $10 \mathrm{wt} \%$ loading does not result in a doubling of the water uptake. Furthermore, adsorption of water increases only slightly from 5 to 10 to $20 \mathrm{wt} \% \mathrm{MOF}$ and is similar for 20 and $40 \mathrm{wt} \%$ films. Bearing the above factors in mind, the water isotherm data at $11 \% \mathrm{RH}$ show a similar trend to the total NO release data expressed per gram of film illustrated in Figure $2 \mathrm{~b}$ and listed in Table S3, where the total quantity of $\mathrm{NO}$ released is greatest (and equal) for films containing 20 and $40 \mathrm{wt} \% \mathrm{MOF}$ and lowest for those containing 5 wt \% MOF. With regard to NO release kinetics, some correlation may be indicated between the overall water adsorption experiment duration and the time taken for the NO release to fall below $30 \mathrm{ppb}$ for each film (see Table S4). In both cases, the length of time increases with MOF wt \%, which again may indicate a more complex gas transport for composite films with higher MOF loadings.

Antibacterial Tests. As mentioned earlier, each particular biological response to $\mathrm{NO}$ requires delivery of a specific concentration. The concentrations released from many of the films analyzed in this study appear to be in the correct region to be antibacterial when compared to results of our previous studies. ${ }^{38}$ To test this assumption, films containing 10 wt \% (i.e. the most efficient formulation) were tested for antimicrobial activity against $P$. aeruginosa. This bacterial species is the most common cause of CAUTIs and is notoriously difficult to treat with antibiotics. It is therefore a good candidate with which to prove the concept. Testing was carried out using a blank PU film, a 10 wt \% CPO-27-Ni-RT composite film, and a $10 \mathrm{wt} \%$ CPO-27-Ni-RT composite film loaded with NO. Antibacterial performance was assessed after 5 and $24 \mathrm{~h}$ incubation periods. This time span is relevant to short-term catheter use, for example, during surgical procedures. It is also applicable to catheters employed over much longer time periods as it is believed that the most critical point in the deployment of a catheter is the initial insertion. At this time, bacteria can easily be carried into the body on the catheter surface and lead to infection. Table S5 lists the obtained cell densities of all samples tested, and the data are illustrated in Figure 8.

Figure 8 shows that during the test periods ( 5 and $24 \mathrm{~h}$ ), bacterial growth was observed in the wells containing $P$. aeruginosa only, blank polymer film, and composite film containing $10 \mathrm{wt} \% \mathrm{CPO}-27-\mathrm{RT}$ without NO. These data suggest that neither the polymer nor MOF imparts antibacterial efficacy toward P. aeruginosa. This is as expected given that the employed polymer is of medical grade and that previously reported studies show low toxicity of this MOF 

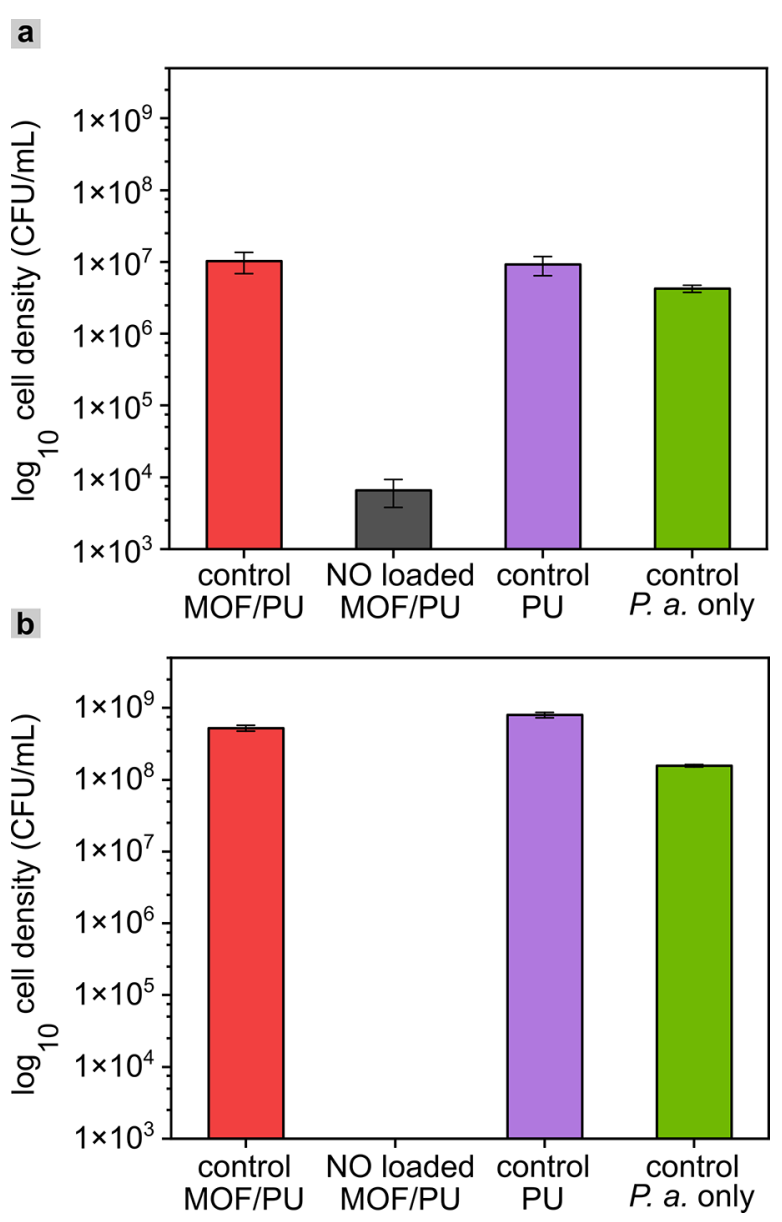

Figure 8. Results of antibacterial testing of CPO-27-Ni-RT-10 wt \% composite (MOF/PU) and CPO-27-Ni-RT-10 wt \% composite NO loaded (NO loaded MOF/PU) against Gram negative bacteria $P$. aeruginosa ATCC 15442 ( $P$. a. only) following incubation times of (a) 5 and (b) $24 \mathrm{~h}$. Error bars represent the standard error of the mean, $N$ $=6$. The control was a blank PU film.

toward human cell lines. ${ }^{38,67}$ Figure 8 a shows that at the short contact time of $5 \mathrm{~h}$, there was sufficient release of NO from the 10 wt \% loaded composite to significantly reduce the bacterial cell density count. At this time-point, a $\log _{10}$ reduction of 2.8 is recorded, which indicates that the surface of the composite is bacteriostatic, that is, the total accumulated release of $\mathrm{NO}$ is inhibiting the growth of the bacteria (note a $\log _{10}$ reduction of 3 is an industrially recognized standard to show antibacterial efficacy). ${ }^{68}$ Further delivery of NO from the surface of the composites leads to a complete destruction of all bacteria, as indicated in Figure $8 \mathrm{~b}(24 \mathrm{~h}$ time period). This equates to a $\log _{10}$ reduction of 8.2 and can be regarded as being bactericidal. The mechanism of action of NO is therefore found to be time-dependent. A similar time-dependent response was observed in our previous studies against $E$. coli and $S$. aureus, though subtle differences exist between the precise responses to each bacteria. These results indicate that the bactericidal activity demonstrated by NO-loaded films is attributable to the released NO. They also confirm for the first time that NO-loaded CPO-27-Ni-RT represents a novel, promising agent for the delivery of biologically relevant concentrations of NO from the surface of PU formulations.

By combining these results with our previously presented antibacterial analysis, bactericidal efficacy of NO-releasing
MOF/PU films has been demonstrated against one Gram positive and two Gram negative bacteria. ${ }^{38}$ These results clearly illustrate the great potential of NO-releasing MOFs for antibacterial applications and provide a valuable proof of concept. Although wider antimicrobial analysis should be conducted (e.g., involving a broader range of bacterial species, fungi and yeast at various and longer time points) to confirm antimicrobial efficacy as part of any end-application development, these results suggest that MOFs could indeed potentially offer an effective solution to catheter-related HAIs.

\section{CONCLUSIONS}

In this study, we have reported a multifaceted investigation of the formulation of $\mathrm{CPO}-27-\mathrm{Ni}$ into a model $\mathrm{PU}$ within the context of NO delivery for medical device applications. Through the analysis of NO release profiles across a range of formulations, we have revealed that the efficiency of $\mathrm{NO}$ release by the MOF changes with wt \% loading and reaches a maximum at 10-20 wt \%. Indeed, the wt \% MOF loading has been shown to be an effective tool to tune both the released quantity and release duration. The analysis of mechanical properties reveals that the composite materials endure remarkable mechanical stress and strain akin to neat PU and that no detrimental effect occurs at loading levels up to $10 \mathrm{wt}$ $\%$. We propose that guest transport is more tortuous in composites containing greater than $10 \mathrm{wt} \% \mathrm{MOF}$, as indicated by the water adsorption analysis, and that this may explain the variation in efficiency with MOF loading and particularly the reduction in efficiency demonstrated by the films containing 40 wt \% MOF. 3D reconstructions of the MOF distribution within the films suggest that extensive connectivity between MOF agglomerates at high loading levels creates a continuous network of MOF particles. This phenomenon may be responsible for the more complex guest transport and may also be the reason why the ductility and toughness of the films are observed to decrease dramatically above 10 wt \%. Therefore, we conclude that the optimum loading level for achieving highest $\mathrm{NO}$ release concentration from this MOF without detriment to polymer properties is $10 \mathrm{wt} \%$. However, as different biological responses are triggered by different concentrations of NO, the wt \% of MOF must be carefully selected depending on the targeted end application. We have shown for the first time through MD simulations and advanced FIB-SEM analysis the excellent compatibility between CPO27-Ni and PU, revealing a strong physical and chemical interaction between the phases and a high degree of pore penetration by the polymer. These findings offer a potential explanation for the attenuation of the MOF NO release performance by the polymer, although an in-depth study of the gas transport of $\mathrm{NO}$ and $\mathrm{H}_{2} \mathrm{O}$ would help to further understand the underlying dynamics. The antibacterial analysis results corroborate our earlier findings that biologically active levels of NO are released from CPO-27-Ni-PU films, even after attenuation by the polymer. Indeed, results confirm that despite the lower surface area exhibited by CPO-27-Ni produced by the water-based room temperature method and the slightly lower levels of NO released by this material, its performance is still more than adequate for a potential end application. This study has focused on CPO-27-Ni and, of course, other MOFs should be analyzed to prove the generality of its findings. Nevertheless, it has revealed the remarkable compatibility of this MOF and PU and many critical factors that contribute to the function of these materials. The findings 
will be pertinent to the further development of NO-releasing MOFs for healthcare applications.

\section{ASSOCIATED CONTENT}

\section{SI Supporting Information}

The Supporting Information is available free of charge at https://pubs.acs.org/doi/10.1021/acsami.0c17937.

Physical Characterization (PXRD, TGA, PSD, BET, and FIB-SEM analysis) of MOF powders and composite films and further specifics of $\mathrm{NO}$ adsorption, water isotherms, antibacterial testing, and computational methods (PDF)

\section{AUTHOR INFORMATION}

\section{Corresponding Author}

Russell E. Morris - School of Chemistry, University of St. Andrews, St. Andrews KY16 9ST, U.K.; Department of Physical and Macromolecular Chemistry, Faculty of Sciences, Charles University, 12843 Prague 2, Czech Republic; Email: rem1@st-andrews.ac.uk

\section{Authors}

Simon M. Vornholt - School of Chemistry, University of St. Andrews, St. Andrews KY16 9ST, U.K.; orcid.org/00000001-9490-3785

Morven J. Duncan - School of Chemistry, University of St. Andrews, St. Andrews KY16 9ST, U.K.

Stewart J. Warrender - School of Chemistry, University of St. Andrews, St. Andrews KY16 9ST, U.K.

Rocio Semino - ICGM, Université de Montpellier, CNRS, ENSCM, Montpellier 75005, France; 이잉.org/00000003-3937-7414

Naseem A. Ramsahye - ICGM, Université de Montpellier, CNRS, ENSCM, Montpellier 75005, France

Guillaume Maurin - ICGM, Université de Montpellier, CNRS, ENSCM, Montpellier 75005, France

Martin W. Smith - Defence Science and Technology Laboratory (Dstl), Salisbury, Wiltshire SP4 0JQ U.K.

Jin-Chong Tan - Department of Engineering Science, University of Oxford, Oxford OX1 3PJ, U.K.; (1) orcid.org/ 0000-0002-5770-408X

David N. Miller - School of Chemistry, University of St. Andrews, St. Andrews KY16 9ST, U.K.

Complete contact information is available at:

https://pubs.acs.org/10.1021/acsami.0c17937

\section{Notes}

The authors declare no competing financial interest.

\section{ACKNOWLEDGMENTS}

S.M.V. would like to thank the EPSRC for funding opportunities under grant agreement EP/K005499/1. S.M.V. and D.N.M. would further like to acknowledge the EPSRC Capital for Great Technologies grant (EP/L017008/1) and the EPSRC Strategic Equipment Resource grant (EP/R023751) for funding and supporting electron microscopy facilities at the University of St Andrews. M.J.D. and S.J.W. would like to acknowledge the ProDIA project that has received funding from the European Union's Horizon 2020 research and innovation programme under grant agreement no. 685727. R.S., N.A.R., and G.M. would like to thank GENCI for granted access to HPC resources of CINES under the allocation DARI
A0080907613. Furthermore, the authors acknowledge Dr Anne-Marie Salisbury at 5D Health Protection Group Ltd. for antibacterial testing.

\section{REFERENCES}

(1) Luo, J.-d.; Chen, A. F. Nitric Oxide: A Newly Discovered Function on Wound Healing. Acta Pharmacol. Sin. 2005, 26, 259264.

(2) Butler, A. R.; Williams, D. L. H. The Physiological Role of Nitric Oxide. Chem. Soc. Rev. 1993, 22, 233.

(3) Ignarro, L. J.; Buga, G. M.; Wood, K. S.; Byrns, R. E.; Chaudhuri, G.; Sawyer, C. H. Endothelium-derived relaxing factor produced and released from artery and vein is nitric oxide. Med. Sci. 1987, 84, 9265-9269.

(4) Jones, M. L.; Ganopolsky, J. G.; Labbé, A.; Wahl, C.; Prakash, S. Antimicrobial Properties of Nitric Oxide and Its Application in Antimicrobial Formulations and Medical Devices. Appl. Microbiol. Biotechnol. 2010, 88, 401-407.

(5) Schairer, D. O.; Chouake, J. S.; Nosanchuk, J. D.; Friedman, A. J. The Potential of Nitric Oxide Releasing Therapies as Antimicrobial Agents. Virulence 2012, 3, 271-279.

(6) Potter, C. F.; Dreshaj, I. A.; Haxhiu, M. A.; Stork, E. K.; Chatburn, R. L.; Martin, R. J. Effect of Exogenous and Endogenous Nitric Oxide on the Airway and Tissue Components of Lung Resistance in the Newborn Piglet. Pediatr. Res. 1997, 41, 886-891.

(7) Moon, W.; Tait, T. A. Controlled Delivery of Medical Gases Using Diffusion Membranes. U.S. Patent 20,160,121,071 A1, 2016.

(8) Thakker, A.; Briggs, N.; Maeda, A.; Byrne, J.; Davey, J. R.; Jackson, T. D. Reducing the Rate of Post-Surgical Urinary Tract Infections in Orthopedic Patients. BMJ Open Qual. 2018, 7, No. e000177.

(9) Furukawa, H.; Cordova, K. E.; O’Keeffe, M.; Yaghi, O. M. The Chemistry and Applications of Metal-Organic Frameworks. Science 2013, 341, 1230444.

(10) Bachman, J. E.; Kapelewski, M. T.; Reed, D. A.; Gonzalez, M. I.; Long, J. R. M2(m-Dobdc) $(\mathrm{M}=\mathrm{Mn}, \mathrm{Fe}, \mathrm{Co}, \mathrm{Ni})$ Metal-Organic Frameworks as Highly Selective, High-Capacity Adsorbents for Olefin/Paraffin Separations. J. Am. Chem. Soc. 2017, 139, 1536315370.

(11) McKinlay, A. C.; Xiao, B.; Wragg, D. S.; Wheatley, P. S.; Megson, I. L.; Morris, R. E. Exceptional Behavior over the Whole Adsorption-Storage-Delivery Cycle for NO in Porous Metal Organic Frameworks. J. Am. Chem. Soc. 2008, 130, 10440-10444.

(12) Zhou, D.-D.; Chen, P.; Wang, C.; Wang, S.-S.; Du, Y.; Yan, H.; Ye, Z.-M.; He, C.-T.; Huang, R.-K.; Mo, Z.-W.; Huang, N.-Y.; Zhang, J.-P. Intermediate-Sized Molecular Sieving of Styrene from Larger and Smaller Analogues. Nat. Mater. 2019, 18, 994-998.

(13) Adil, K.; Belmabkhout, Y.; Pillai, R. S.; Cadiau, A.; Bhatt, P. M.; Assen, A. H.; Maurin, G.; Eddaoudi, M. Gas/Vapour Separation Using Ultra-Microporous Metal-Organic Frameworks: Insights into the Structure/Separation Relationship. Chem. Soc. Rev. 2017, 46, $3402-3430$

(14) Palomba, J. M.; Credille, C. V.; Kalaj, M.; DeCoste, J. B.; Peterson, G. W.; Tovar, T. M.; Cohen, S. M. High-Throughput Screening of Solid-State Catalysts for Nerve Agent Degradation. Chem. Commun. 2018, 54, 5768-5771.

(15) Tuttle, R. R.; Rubin, H. N.; Rithner, C. D.; Finke, R. G.; Reynolds, M. M. Copper Ion vs Copper Metal-Organic Framework Catalyzed NO Release from Bioavailable S-Nitrosoglutathione En Route to Biomedical Applications: Direct 1H NMR Monitoring in Water Allowing Identification of the Distinct, True Reaction Stoichiometries and Thio. J. Inorg. Biochem. 2019, 199, 110760.

(16) Shiozawa, H.; Bayer, B. C.; Peterlik, H.; Meyer, J. C.; Lang, W.; Pichler, T. Doping of Metal-Organic Frameworks towards Resistive Sensing. Sci. Rep. 2017, 7, 2439.

(17) Wannapaiboon, S.; Schneemann, A.; Hante, I.; Tu, M.; Epp, K.; Semrau, A. L.; Sternemann, C.; Paulus, M.; Baxter, S. J.; Kieslich, G.; Fischer, R. A. Control of Structural Flexibility of Layered-Pillared 
Metal-Organic Frameworks Anchored at Surfaces. Nat. Commun. 2019, 10, 346.

(18) McKinlay, A. C.; Morris, R. E.; Horcajada, P.; Férey, G.; Gref, R.; Couvreur, P.; Serre, C. BioMOFs: Metal-Organic Frameworks for Biological and Medical Applications. Angew. Chem. Int. Ed. 2010, 49, 6260-6266.

(19) AL Haydar, M.; Abid, H.; Sunderland, B.; Wang, S. Metal Organic Frameworks as a Drug Delivery System for Flurbiprofen. Drug Des., Dev. Ther. 2017, 11, 2685-2695.

(20) Chowdhury, M. A. Metal-Organic-Frameworks for Biomedical Applications in Drug Delivery, and as MRI Contrast Agents. J. Biomed. Mater. Res., Part A 2017, 105, 1184-1194.

(21) Hibbard, H. A. J.; Reynolds, M. M. Fluorescent Nitric Oxide Donor for the Detection and Killing of Pseudomonas Aeruginosa. J. Mater. Chem. B 2019, 7, 2009-2018.

(22) Horcajada, P.; Gref, R.; Baati, T.; Allan, P. K.; Maurin, G.; Couvreur, P.; Férey, G.; Morris, R. E.; Serre, C. Metal-Organic Frameworks in Biomedicine. Chem. Rev. 2012, 112, 1232-1268.

(23) Cattaneo, D.; Warrender, S. J.; Duncan, M. J.; Kelsall, C. J.; Doherty, M. K.; Whitfield, P. D.; Megson, I. L.; Morris, R. E. Tuning the Nitric Oxide Release from CPO-27 MOFs. RSC Adv. 2016, 6, 14059-14067.

(24) McKinlay, A. C.; Allan, P. K.; Renouf, C. L.; Duncan, M. J.; Wheatley, P. S.; Warrender, S. J.; Dawson, D.; Ashbrook, S. E.; Gil, B.; Marszalek, B.; Düren, T.; Williams, J. J.; Charrier, C.; Mercer, D. K.; Teat, S. J.; Morris, R. E. Multirate Delivery of Multiple Therapeutic Agents from Metal-Organic Frameworks. APL Mater. 2014, 2, 124108-124115.

(25) Pinto, R. V.; Wang, S.; Tavares, S. R.; Pires, J.; Antunes, F.; Vimont, A.; Clet, G.; Daturi, M.; Maurin, G.; Serre, C.; Pinto, M. L. Tuning Cellular Biological Functions Through the Controlled Release of NO from a Porous Ti-MOF. Angew. Chem., Int. Ed. 2020, 59, 5135-5143.

(26) Neufeld, B. H.; Neufeld, M. J.; Lutzke, A.; Schweickart, S. M.; Reynolds, M. M. Metal-Organic Framework Material Inhibits Biofilm Formation of Pseudomonas Aeruginosa. Adv. Funct. Mater. 2017, 27, 1702255.

(27) Senkovska, I.; Cychosz, K. A.; Llewellyn, P.; Thommes, M.; Kaskel, S. The Chemistry of Metal-Organic Frameworks: Synthesis, Characterization, and Applications. In The Chemistry of MetalOrganic Frameworks: Synthesis, Characterization, and Applications; Kaskel, S., Ed.; Wiley-VCH Verlag GmbH \& Co. KGaA: Weinheim, Germany, 2016; pp 575-605.

(28) Rieth, A. J.; Wright, A. M.; Dincă, M. Kinetic Stability of Metal-Organic Frameworks for Corrosive and Coordinating Gas Capture. Nat. Rev. Mater. 2019, 4, 708-725.

(29) Rosi, N. L.; Kim, J.; Eddaoudi, M.; Chen, B.; O’Keeffe, M.; Yaghi, O. M. Rod Packings and Metal-Organic Frameworks Constructed from Rod-Shaped Secondary Building Units. J. Am. Chem. Soc. 2005, 127, 1504-1518.

(30) Dietzel, P. D. C.; Morita, Y.; Blom, R.; FjellvÅg, H. An In Situ High-Temperature Single-Crystal Investigation of a Dehydrated Metal-Organic Framework Compound and Field-Induced Magnetization of One-Dimensional Metal-Oxygen Chains. Angew. Chem., Int. Ed. 2005, 44, 6354-6358.

(31) Rosnes, M. H.; Pato-Doldán, B.; Johnsen, R. E.; Mundstock, A.; Caro, J.; Dietzel, P. D. C. Role of the Metal Cation in the Dehydration of the Microporous Metal-Organic Frameworks CPO-27-M. Microporous Mesoporous Mater. 2020, 309, 110503.

(32) Lowe, A.; Chittajallu, P.; Gong, Q.; Li, J.; Balkus, K. J. Storage and Delivery of Nitric Oxide via Diazeniumdiolated Metal Organic Framework. Microporous Mesoporous Mater. 2013, 181, 17-22.

(33) Jen, M. C.; Serrano, M. C.; van Lith, R.; Ameer, G. A. PolymerBased Nitric Oxide Therapies: Recent Insights for Biomedical Applications. Adv. Funct. Mater. 2012, 22, 239-260.

(34) Pant, J.; Goudie, M. J.; Chaji, S. M.; Johnson, B. W.; Handa, H. Nitric Oxide Releasing Vascular Catheters for Eradicating Bacterial Infection. J. Biomed. Mater. Res., Part B 2018, 106, 2849-2857.
(35) Homeyer, K. H.; Goudie, M. J.; Singha, P.; Handa, H. LiquidInfused Nitric-Oxide-Releasing Silicone Foley Urinary Catheters for Prevention of Catheter-Associated Urinary Tract Infections. ACS Biomater. Sci. Eng. 2019, 5, 2021-2029.

(36) Choi, M.; Park, S.; Park, K.; Jeong, H.; Hong, J. Nitric Oxide Delivery Using Biocompatible Perfluorocarbon Microemulsion for Antibacterial Effect. ACS Biomater. Sci. Eng. 2019, 5, 1378-1383.

(37) Urzedo, A. L.; Gonçalves, M. C.; Nascimento, M. H. M.; Lombello, C. B.; Nakazato, G.; Seabra, A. B. Multifunctional Alginate Nanoparticles Containing Nitric Oxide Donor and Silver Nanoparticles for Biomedical Applications. Mater. Sci. Eng., C 2020, 112, 110933.

(38) Duncan, M. J.; Wheatley, P. S.; Coghill, E. M.; Vornholt, S. M.; Warrender, S. J.; Megson, I. L.; Morris, R. E. Antibacterial Efficacy from NO-Releasing MOF-Polymer Films. Adv. Mater. 2020, 1, 2509-2519.

(39) Kalaj, M.; Bentz, K. C.; Ayala, S.; Palomba, J. M.; Barcus, K. S.; Katayama, Y.; Cohen, S. M. MOF-Polymer Hybrid Materials: From Simple Composites to Tailored Architectures. Chem. Rev. 2020, 120, $8267-8302$

(40) Denny, M. S.; Moreton, J. C.; Benz, L.; Cohen, S. M. MetalOrganic Frameworks for Membrane-Based Separations. Nat. Rev. Mater. 2016, 1, 16078.

(41) Lin, R.; Villacorta Hernandez, B.; Ge, L.; Zhu, Z. Metal Organic Framework Based Mixed Matrix Membranes: An Overview on Filler/ Polymer Interfaces. J. Mater. Chem. A 2018, 6, 293-312.

(42) Cattaneo, D.; Warrender, S. J.; Duncan, M. J.; Castledine, R.; Parkinson, N.; Haley, I.; Morris, R. E. Water Based Scale-up of CPO27 Synthesis for Nitric Oxide Delivery. Dalton Trans. 2016, 45, 618629 .

(43) Rouquerol, J.; Llewellyn, P.; Rouquerol, F. Is the BET Equation Applicable to Microporous Adsorbents? Stud. Surf. Sci. Catal. 2007, $160,49-56$.

(44) Semino, R.; Ramsahye, N. A.; Ghoufi, A.; Maurin, G. Microscopic Model of the Metal-Organic Framework/Polymer Interface: A First Step toward Understanding the Compatibility in Mixed Matrix Membranes. ACS Appl. Mater. Interfaces 2016, 8, 809819.

(45) Perdew, J. P.; Burke, K.; Ernzerhof, M. Generalized Gradient Approximation Made Simple. Phys. Rev. Lett. 1996, 77, 3865-3868.

(46) Grimme, S. Semiempirical GGA-Type Density Functional Constructed with a Long-Range Dispersion Correction. J. Comput. Chem. 2006, 27 (15), 1787-1799.

(47) Hutter, J.; Iannuzzi, M.; Schiffmann, F.; VandeVondele, J. Cp2k: Atomistic Simulations of Condensed Matter Systems. Wiley Interdiscip. Rev.: Comput. Mol. Sci. 2014, 4, 15-25.

(48) Abbott, L. J.; Hart, K. E.; Colina, C. M. Polymatic: A Generalized Simulated Polymerization Algorithm for Amorphous Polymers. Theor. Chem. Acc. 2013, 132, 1334.

(49) Eggimann, B. L.; Sunnarborg, A. J.; Stern, H. D.; Bliss, A. P.; Siepmann, J. I. An Online Parameter and Property Database for the TraPPE Force Field. Mol. Simul. 2014, 40, 101-105.

(50) Bayly, C. I.; Cieplak, P.; Cornell, W.; Kollman, P. A. A WellBehaved Electrostatic Potential Based Method Using Charge Restraints for Deriving Atomic Charges: The RESP Model. J. Phys. Chem. 1993, 97, 10269-10280.

(51) Wang, J.; Wolf, R. M.; Caldwell, J. W.; Kollman, P. A.; Case, D. A. Development and Testing of a General Amber Force Field. J. Comput. Chem. 2004, 25, 1157-1174.

(52) Todorov, I. T.; Smith, W.; Trachenko, K.; Dove, M. T. DL_POLY_3: New Dimensions in Molecular Dynamics Simulations via Massive Parallelism. J. Mater. Chem. 2006, 16, 1911.

(53) Rappe, A. K.; Casewit, C. J.; Colwell, K. S.; Goddard, W. A.; Skiff, W. M. UFF, a Full Periodic Table Force Field for Molecular Mechanics and Molecular Dynamics Simulations. J. Am. Chem. Soc. 1992, 114, 10024-10035.

(54) Mayo, S. L.; Olafson, B. D.; Goddard, W. A. DREIDING: A Generic Force Field for Molecular Simulations. J. Phys. Chem. 1990, 94, 8897-8909. 
(55) Zheng, J.; Barpaga, D.; Trump, B. A.; Shetty, M.; Fan, Y.; Bhattacharya, P.; Jenks, J. J.; Su, C.-Y.; Brown, C. M.; Maurin, G.; McGrail, B. P.; Motkuri, R. K. Molecular Insight into Fluorocarbon Adsorption in Pore Expanded Metal-Organic Framework Analogs. J. Am. Chem. Soc. 2020, 142, 3002-3012.

(56) Mahdi, E. M.; Tan, J.-C. Mixed-Matrix Membranes of Zeolitic Imidazolate Framework (ZIF-8)/Matrimid Nanocomposite: ThermoMechanical Stability and Viscoelasticity Underpinning Membrane Separation Performance. J. Membr. Sci. 2016, 498, 276-290.

(57) Dietzel, P. D. C.; Johnsen, R. E.; Blom, R.; FjellvÅg, H. Structural Changes and Coordinatively Unsaturated Metal Atoms on Dehydration of Honeycomb Analogous Microporous Metal-Organic Frameworks. Chem. - Eur. J. 2008, 14, 2389-2397.

(58) Dechnik, J.; Sumby, C. J.; Janiak, C. Enhancing Mixed-Matrix Membrane Performance with Metal-Organic Framework Additives. Cryst. Growth Des. 2017, 17, 4467-4488.

(59) Semino, R.; Dürholt, J. P.; Schmid, R.; Maurin, G. Multiscale Modeling of the HKUST-1/Poly(Vinyl Alcohol) Interface: From an Atomistic to a Coarse Graining Approach. J. Phys. Chem. C 2017, 121, 21491-21496.

(60) Bonino, F.; Chavan, S.; Vitillo, J. G.; Groppo, E.; Agostini, G.; Lamberti, C.; Dietzel, P. D. C.; Prestipino, C.; Bordiga, S. Local Structure of CPO-27-Ni Metallorganic Framework upon Dehydration and Coordination of NO. Chem. Mater. 2008, 20, 4957-4968.

(61) Zhang, Y.; Feng, X.; Yuan, S.; Zhou, J.; Wang, B. Challenges and Recent Advances in MOF-Polymer Composite Membranes for Gas Separation. Inorg. Chem. Front. 2016, 3, 896-909.

(62) Chen, X.; Song, S.; Wang, L.; Zhang, X.; Fu, D.; Zhang, G. Ultrathin Metal-Organic Framework Membranes Used for Industrial Separation. IOP Conf. Ser. Earth Environ. Sci. 2018, 170, 052040.

(63) Dong, G.; Li, H.; Chen, V. Challenges and Opportunities for Mixed-Matrix Membranes for Gas Separation. J. Mater. Chem. A 2013, 1,4610 .

(64) Ahmadi, M.; Janakiram, S.; Dai, Z.; Ansaloni, L.; Deng, L. Performance of Mixed Matrix Membranes Containing Porous TwoDimensional (2D) and Three-Dimensional (3D) Fillers for CO2 Separation: A Review. Membranes 2018, 8, 50.

(65) Semino, R.; Moreton, J. C.; Ramsahye, N. A.; Cohen, S. M.; Maurin, G. Understanding the Origins of Metal-Organic Framework/Polymer Compatibility. Chem. Sci. 2018, 9, 315-324.

(66) Smith, Z. P.; Bachman, J. E.; Li, T.; Gludovatz, B.; Kusuma, V. A.; Xu, T.; Hopkinson, D. P.; Ritchie, R. O.; Long, J. R. Increasing M 2 (Dobdc) Loading in Selective Mixed-Matrix Membranes: A Rubber Toughening Approach. Chem. Mater. 2018, 30, 1484-1495.

(67) Ruyra, A.; Yazdi, A.; Espín, J.; Carné-Sánchez, A.; Roher, N.; Lorenzo, J.; Imaz, I.; Maspoch, D. Synthesis, Culture Medium Stability, and In Vitro and In Vivo Zebrafish Embryo Toxicity of Metal-Organic Framework Nanoparticles. Chem.-Eur. J. 2015, 21, $2508-2518$.

(68) ISO 22196:2011-Measurement of antibacterial activity on plastics and other non-porous surfaces. https://www.iso.org/ standard/54431.html (accessed November 20, 2020). 